\title{
Elliptic gamma-function and multi-spin solutions of the Yang-Baxter equation
}

\author{
Vladimir V. Bazhanov ${ }^{\mathrm{a}, \mathrm{b}, *}$, Sergey M. Sergeev ${ }^{\mathrm{c}}$ \\ a Department of Theoretical Physics, Research School of Physics and Engineering, Australian National University, \\ Canberra, ACT 0200, Australia \\ b Mathematical Sciences Institute, Australian National University, Canberra, ACT 0200, Australia \\ ${ }^{\mathrm{c}}$ Faculty of Information Sciences and Engineering, University of Canberra, Bruce, ACT 2601, Australia
}

Received 18 September 2011; accepted 27 October 2011

Available online 6 November 2011

\begin{abstract}
We present a generalization of the master solution to the quantum Yang-Baxter equation (obtained recently in arXiv:1006.0651) to the case of multi-component continuous spin variables taking values on a circle. The Boltzmann weights are expressed in terms of the elliptic gamma-function. The associated solvable lattice model admits various equivalent descriptions, including an interaction-round-a-face formulation with positive Boltzmann weights. In the quasi-classical limit the model leads to a new series of classical discrete integrable equations on planar graphs.
\end{abstract}

(c) 2011 Published by Elsevier B.V.

\section{Introduction}

There are solvable lattice models of statistical mechanics with only a pair interaction between neighbouring spins, i.e., where two spins interact only if they are connected by an edge of the lattice. The Yang-Baxter equation for these models usually takes the form of the "star-triangle relation" [1]. The most notable discrete-spin models in this class include the Kashiwara-Miwa [2] and chiral Potts [3-5] models (both of them also contain the Ising model [1] and FateevZamolodchikov $Z_{N}$-model [6] as particular cases) see [7] for a review. There are also important

\footnotetext{
* Corresponding author at: Department of Theoretical Physics, Research School of Physics and Engineering, Australian National University, Canberra, ACT 0200, Australia.

E-mail addresses: Vladimir.Bazhanov@anu.edu.au (V.V. Bazhanov), Sergey.Sergeev@ canberra.edu.au (S.M. Sergeev).
} 
continuous spin models, including Zamolodchikov's "fishing-net" model [8], which describes certain planar Feynman diagrams in quantum field theory, and the Faddeev-Volkov model [9], connected with quantization [10] of discrete conformal transformations [11,12].

Recently [13] we have found a new solution of the star-triangle relation which contains as special cases all the discrete- and continuous-spin solutions mentioned above, ${ }^{1}$ and also leads to new ones. This "master solution" is expressed through elliptic gamma-functions and contains two temperature-like variables. It defines an exactly solvable lattice models with continuous spin variables taking values on a circle. Its connection to the theory of elliptic hypergeometric functions is discussed in [14]. From an algebraic point of view the model is related to the modular double $[15,16]$ of the Sklyanin algebra [17]. The latter is an elliptic deformation of the quantum group $U_{q}(s l(2))$, connected with the $R$-matrix of the eight-vertex model [18].

In this paper we extend the main results of [13] to the case related to the $s l(n)$ algebra with $n \geqslant 3$. The generalized model has multi-component continuous spin variables taking values on a circle. Similarly to the $n=2$ case of [13] the model contains two temperature-like parameters. The Boltzmann weights satisfy the so-called star-star relation $[19,20]$ (see Eq. (26) below), which ensures the integrability of the model. Currently, we claim this relation as a conjecture, however, we expect that a complete proof could be obtained by a rather straightforward generalization of the results of [20-24] devoted to discrete-spin models connected with the $s l(n)$ algebra.

It should be noted, that apart from the $n=2$ case previously considered in [13], the two-spin Boltzmann weights are not real and positive. Fortunately, this is not an indication that the model is unphysical. It can be reformulated as an "interaction-round-a-face" (IRF) model and then there exist a domain of parameters, where the IRF-type Boltzmann weights become real and positive, see Section 3.

The quasi-classical (or low-temperature) limit of the model is considered in Section 4. A stationary spin configuration which gives the leading contribution to the partition function in this limit is described by new classical discrete integrable equations for multi-component fields assigned to lattice sites. These equations can be thought as a generalization of the Laplace-type equation associated with the famous $Q_{4}$ system [25,26] to the multi-component case.

In Section 5 we summarize the results and discuss their connections with some other integrable systems in two and three dimensions.

\section{Formulation of the model}

We start with the definition of the elliptic gamma-function [16,27-29]. Let $q$, $p$ be two elliptic nomes (they play the role of the temperature-like parameters),

$$
\mathrm{p}=\mathrm{e}^{\mathrm{i} \pi \sigma}, \quad \mathrm{q}=\mathrm{e}^{\mathrm{i} \pi \tau}, \quad \operatorname{Im} \sigma>0, \quad \operatorname{Im} \tau>0 .
$$

In principle, these parameters can be arbitrary (apart from the requirements $|p|<1$ and $|q|<1$ ), however, in the following we will often refer to special regimes when $p$ and $q$ are either real or complex conjugate to each other,

$$
\text { (i) } \mathrm{p}^{*}=\mathrm{p}, \quad \mathrm{q}^{*}=\mathrm{q}, \quad \text { (ii) } \mathrm{p}^{*}=\mathrm{q} \text {. }
$$

Note that in both of these cases the "crossing parameter"

\footnotetext{
1 To be more precise, it only contains the solutions, which have a single one-dimensional spin at each lattice site. For this reason, it cannot contain the $D \geqslant 2$ fishing-net model which has multi-dimensional spins.
} 


$$
\eta=-\mathrm{i} \pi(\sigma+\tau) / 2,
$$

is real and positive. Define the elliptic gamma-function ${ }^{2}$

$$
\Phi(z)=\prod_{j, k=0}^{\infty} \frac{1-\mathrm{e}^{2 \mathrm{i} z} \mathrm{q}^{2 j+1} \mathrm{p}^{2 k+1}}{1-\mathrm{e}^{-2 \mathrm{i} z} \mathrm{q}^{2 j+1} \mathrm{p}^{2 k+1}}=\exp \left\{\sum_{k \neq 0} \frac{\mathrm{e}^{-2 \mathrm{i} z k}}{k\left(\mathrm{q}^{k}-\mathrm{q}^{-k}\right)\left(\mathrm{p}^{k}-\mathrm{p}^{-k}\right)}\right\},
$$

where the product formula is valid for all $z$, while the exponential formula is only valid in the strip

$$
-\operatorname{Re} \eta<\operatorname{Im} z<\operatorname{Re} \eta
$$

The function (4) possesses simple periodicity and "reflection" properties

$$
\Phi(z+\pi)=\Phi(z), \quad \Phi(z) \Phi(-z)=1 .
$$

Moreover, it satisfies the following difference equation

$$
\frac{\Phi\left(z-\frac{\pi \sigma}{2}\right)}{\Phi\left(z+\frac{\pi \sigma}{2}\right)}=\prod_{n=0}^{\infty}\left(1-\mathrm{e}^{2 \mathrm{i} z} \mathrm{q}^{2 n+1}\right)\left(1-\mathrm{e}^{-2 \mathrm{i} z} \mathrm{q}^{2 n+1}\right)=\bar{\vartheta}_{4}(z \mid \tau)
$$

and a similar equation obtained by interchanging $\tau$ and $\sigma$. Here

$$
\bar{\vartheta}_{j}(z \mid \tau)=\frac{1}{G(\tau)} \vartheta_{j}(z \mid \tau), \quad G(\tau)=\prod_{k=1}^{\infty}\left(1-\mathrm{q}^{2 k}\right), \mathrm{q}=\mathrm{e}^{\mathrm{i} \pi \tau},
$$

where $\vartheta_{j}(z \mid \tau), j=1,2,3,4$, stand for the standard elliptic theta-functions of the periods $\pi$ and $\pi \tau$, as defined in [30]. Note also, than in the regimes (2) the function (4) has a simple complex conjugation property,

$$
\Phi(z)^{*}=\Phi\left(-z^{*}\right) .
$$

Next, we want to introduce a new two-dimensional solvable edge-interaction model. The model can be formulated on rather general planar graphs, however, for the purposes of this presentation it is convenient to take a regular square lattice. Consider the square lattice, drawn diagonally as in Fig. 1. The edges of the lattice are shown with bold lines and the sites are shown with either open or filled circles in a checkerboard order. In this section we will not distinguish the two type sites; their difference will be important in Section 3. At each lattice site place a $n$-component continuous spin variable

$$
\boldsymbol{x}=\left\{x_{1}, \ldots, x_{n}\right\} \in \mathbb{R}^{n}, \quad 0 \leqslant x_{j}<\pi, \quad \sum_{j=1}^{n} x_{j}=0 \quad(\bmod \pi) .
$$

Note that due to the restriction on the total sum, there are only $(n-1)$ independent variables $x_{j}$. For further reference define the integration measure

$$
\int d \boldsymbol{x}=\int_{0}^{\pi} \cdots \int_{0}^{\pi} d x_{1} \cdots d x_{n-1}, \quad \mathbb{1}=\int|\boldsymbol{x}\rangle d \boldsymbol{x}\langle\boldsymbol{x}| .
$$

\footnotetext{
2 Our function $\Phi(z)$ coincides with $\Gamma\left(\mathrm{e}^{-2 \mathrm{i}(z-\eta)} ; \mathrm{p}^{2}, \mathrm{q}^{2}\right)$ in the notation of Ref. [16]. The definition (4) differs slightly from that of Ref. [13], where the RHS of (4) is denoted as $\Phi(2 z)$.
} 


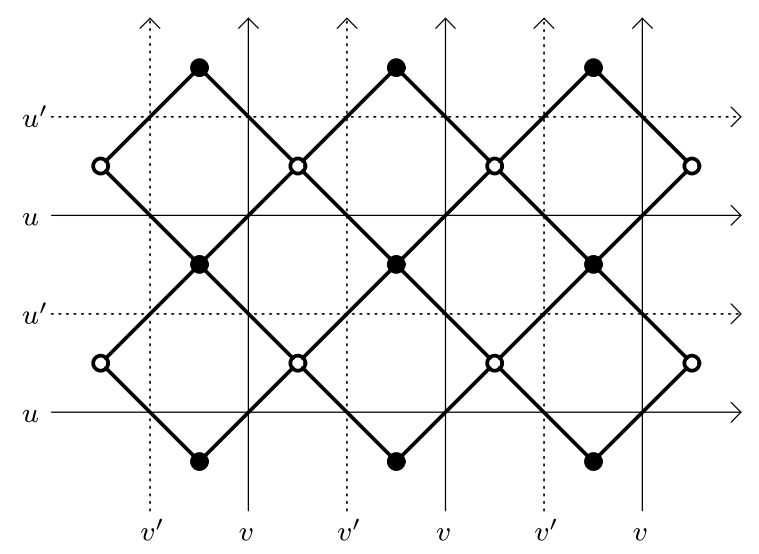

Fig. 1. The square lattice shown with bold sites and bold edges drawn diagonally. The associated medial lattice is drawn with thin and dotted horizontal and vertical lines. The lines are oriented and carry rapidity variables $u, u^{\prime}, v$ and $v^{\prime}$.

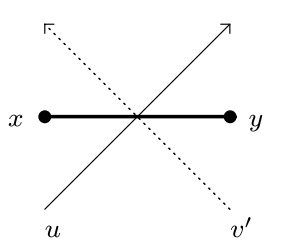

$\mathbb{W}_{u-v^{\prime}}(\boldsymbol{x}, \boldsymbol{y})$

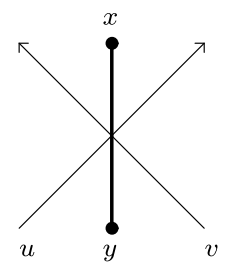

$\overline{\mathbb{W}}_{u-v}(\boldsymbol{y}, \boldsymbol{x})$

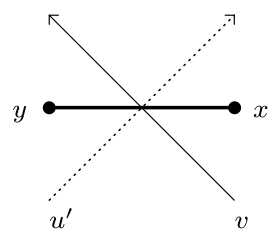

$\mathbb{W}_{u^{\prime}-v}(\boldsymbol{x}, \boldsymbol{y})$

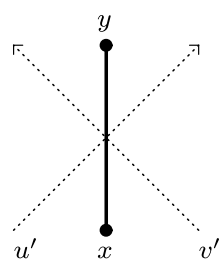

$\overline{\mathbb{W}}_{u^{\prime}-v^{\prime}}(\boldsymbol{y}, \boldsymbol{x})$

Fig. 2. Four different types of edges and their Boltzmann weights.

Fig. 1 also shows an auxiliary medial graph whose sites lie on the edges of the original square lattice. The medial graph is drawn with alternating thin and dotted lines. The lines are directed as indicated by arrows. To each horizontal (vertical) line on the medial graph assign a rapidity variable $u(v)$. In general these variables may be different for different lines. However, a convenient level of generality that we shall use here is to assign the same rapidity $u$ to all thin horizontal lines and the same variable $u^{\prime}$ to all dotted horizontal lines. Similarly, assign the variables $v$ and $v^{\prime}$ to thin and dotted vertical lines as indicated in Fig. 1.

Two spins interact only if they are connected with an edge. The corresponding Boltzmann weight depends on spins at the ends of the edge and on two rapidities passing through the edge. There are four types of edges differing by orientations and types of the directed rapidity lines passing through the edge. They are assigned with different Boltzmann weights as shown in Fig. 2. The weights are defined as

$$
\begin{aligned}
& \mathbb{W}_{\alpha}(\boldsymbol{x}, \boldsymbol{y})=\kappa_{n}(\alpha)^{-1} \prod_{j, k=1}^{n} \Phi\left(x_{j}-y_{k}+\mathrm{i} \alpha\right), \\
& \overline{\mathbb{W}}_{\alpha}(\boldsymbol{x}, \boldsymbol{y})=\sqrt{\mathbb{S}(\boldsymbol{x}) \mathbb{S}(\boldsymbol{y})} \mathbb{W}_{\eta-\alpha}(x, y),
\end{aligned}
$$

where the single-spin function $\mathbb{S}$ is given by 


$$
\begin{aligned}
\mathbb{S}(\boldsymbol{x}) & =\varkappa_{s}^{-1} \prod_{j \neq k}\left\{\Phi\left(x_{j}-x_{k}+\mathrm{i} \eta\right)\right\}^{-1} \\
& =\varkappa_{s}^{-1} \prod_{j<k}\left\{\mathrm{e}^{\eta / 2} \bar{\vartheta}_{1}\left(x_{j}-x_{k} \mid \tau\right) \bar{\vartheta}_{1}\left(x_{j}-x_{k} \mid \sigma\right)\right\}
\end{aligned}
$$

and

$$
\varkappa_{s}=n !\left(\frac{\pi}{G(\tau) G(\sigma)}\right)^{n-1} .
$$

Here the indices $j, k$ run over the values $1,2, \ldots, n$; the functions $\bar{\vartheta}(x \mid \tau)$ and $G(\tau)$ are defined in (8). The normalization factor

$$
\kappa_{n}(\alpha)=\exp \left\{\sum_{k \neq 0} \frac{\mathrm{e}^{2 n k \alpha}}{k\left(\mathrm{p}^{k}-\mathrm{p}^{-k}\right)\left(\mathrm{q}^{k}-\mathrm{q}^{-k}\right)} \frac{\mathrm{p}^{k} \mathrm{q}^{k}-\mathrm{p}^{-k} \mathrm{q}^{-k}}{\mathrm{p}^{n k} \mathrm{q}^{n k}-\mathrm{p}^{-n k} \mathrm{q}^{-n k}}\right\},
$$

has the meaning of the partition function per edge for unnormalized Boltzmann weights (i.e., when the factor $\kappa_{n}(\alpha)$ in (12) is omitted). It solves a pair of functional equations

$$
\kappa_{n}(\alpha) \kappa_{n}(-\alpha)=1, \quad \kappa_{n}(\eta-\alpha) \kappa_{n}(\eta+\alpha)=\Phi(\mathrm{i} \eta-\mathrm{i} n \alpha) \Phi(\mathrm{i} \eta+\mathrm{i} n \alpha) .
$$

The weights (12) satisfy two inversion relations:

$$
\mathbb{W}_{\alpha}(\boldsymbol{x}, \boldsymbol{y}) \mathbb{W}_{-\alpha}(\boldsymbol{y}, \boldsymbol{x})=1, \quad \int d \boldsymbol{x} \overline{\mathbb{W}}_{\alpha}(\boldsymbol{x}, \boldsymbol{z}) \overline{\mathbb{W}}_{-\alpha}(\boldsymbol{z}, \boldsymbol{y})=\frac{1}{n !} \sum_{\hat{\sigma}} \delta(\boldsymbol{x}, \hat{\sigma}(\boldsymbol{y}))
$$

where the sum is taken over $n$ ! permutations $\hat{\sigma}$ of components of the vector $\boldsymbol{y}=\left\{y_{1}, \ldots, y_{n}\right\}$ and the $\delta$-function is understood with respect to the measure (11). The first of these relations is a trivial corollary of the definition (12) and the reflection property of the elliptic gamma-function $\Phi(z)$. The second relation is a particular case of a more general relation stated in Theorem 11 in Ref. [16], see Appendix A for further details.

Note that for any permutation $\hat{\sigma}$ one has

$$
\mathbb{W}(\boldsymbol{x}, \boldsymbol{y})=\mathbb{W}_{\alpha}(\hat{\sigma}(\boldsymbol{x}), \boldsymbol{y})=\mathbb{W}(\boldsymbol{x}, \hat{\sigma}(\boldsymbol{y})), \quad \mathbb{S}(\boldsymbol{x})=\mathbb{S}(\hat{\sigma}(\boldsymbol{x})),
$$

as a trivial consequence of the definitions (12) and (13). Under the complex conjugation the weights transform as

$$
\mathbb{W}_{\alpha}(\boldsymbol{x}, \boldsymbol{y})^{*}=\mathbb{W}_{\alpha^{*}}(\boldsymbol{y}, \boldsymbol{x})=\mathbb{W}_{\alpha^{*}}(-\boldsymbol{x},-\boldsymbol{y}), \quad \mathbb{S}(\boldsymbol{x})^{*}=\mathbb{S}(\boldsymbol{x}),
$$

provided the nomes $p$ and $q$ belong to either of the regimes, defined in (2) (remind that the spin variables are always assumed to be real; see (10)). When $n=2$ the weights $\mathbb{W}$ and $\mathbb{S}$ are real and positive [13]. Correspondingly, the weights $\mathbb{W}_{\alpha}(\boldsymbol{x}, \boldsymbol{y})$ and $\overline{\mathbb{W}}_{\alpha}(\boldsymbol{x}, \boldsymbol{y})$ are symmetric under an exchange of the spins $\boldsymbol{x}$ and $\boldsymbol{y}$. However, for $n>2$ this symmetry is lost. As a particular consequence of this fact the above description of the associated lattice model required two types of the rapidity lines.

The square lattice in Fig. 1 can be formed by periodic translations of the "box diagram" shown in Fig. 3. The Boltzmann weight of this box can be conveniently associated with an $R$-matrix,

$$
\left\langle\boldsymbol{x}, \boldsymbol{y}\left|\mathbb{R}_{\boldsymbol{u} v}\right| \boldsymbol{x}^{\prime}, \boldsymbol{y}^{\prime}\right\rangle=\overline{\mathbb{W}}_{u-v}\left(\boldsymbol{y}, \boldsymbol{x}^{\prime}\right) \overline{\mathbb{W}}_{u^{\prime}-v^{\prime}}\left(\boldsymbol{y}^{\prime}, \boldsymbol{x}\right) \mathbb{W}_{u^{\prime}-v}\left(\boldsymbol{x}^{\prime}, \boldsymbol{y}^{\prime}\right) \mathbb{W}_{u-v^{\prime}}(\boldsymbol{x}, \boldsymbol{y}),
$$

where $\boldsymbol{u}=\left[u, u^{\prime}\right]$ and $\boldsymbol{q}=\left[v, v^{\prime}\right]$ stand for the pairs of rapidities in horizontal and vertical directions. The partition function is defined as 


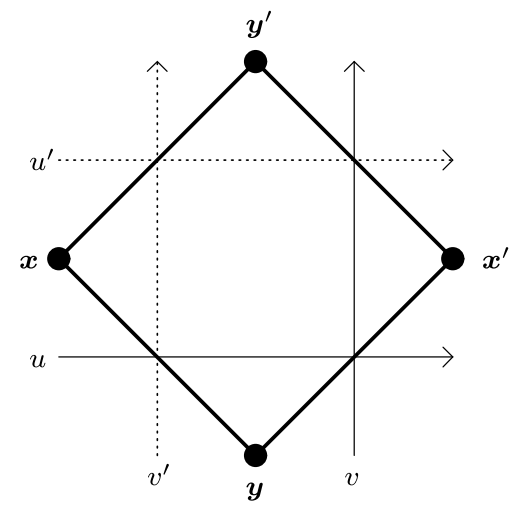

Fig. 3. Pictorial representation of the $R$-matrix $\left\langle\boldsymbol{x}, \boldsymbol{y}\left|R_{\boldsymbol{u} v}\right| \boldsymbol{x}^{\prime}, \boldsymbol{y}^{\prime}\right\rangle$.

$$
Z=\int \prod_{\text {boxes }}\left\langle\boldsymbol{x}, \boldsymbol{y}\left|\mathbb{R}_{\boldsymbol{u v}}\right| \boldsymbol{x}^{\prime}, \boldsymbol{y}^{\prime}\right\rangle \prod_{\text {sites }} d \boldsymbol{x},
$$

where the first product is taken over all boxes and $\boldsymbol{x}, \boldsymbol{y}, \boldsymbol{x}^{\prime}, \boldsymbol{y}^{\prime}$ are the spins at the corners arranged as in Fig. 3. The integral is taken over all configurations of the spin variables on the internal lattice sites. The boundary spins are kept fixed. Note that due to periodicity (6) the definition (21) only contains closed contour integrals (the contours can be deformed into the complex plane, if necessary).

\section{Yang-Baxter equations and star-star relations}

The $R$-matrix (20) can be regarded as a kernel of an integral operator acting on a pair of (multi-component) continuous spin variables (10). We claim that it satisfies the Yang-Baxter equation of the form

$$
\begin{aligned}
& \int d \boldsymbol{x}^{\prime} d \boldsymbol{y}^{\prime} d \boldsymbol{z}^{\prime}\left\langle\boldsymbol{x}, \boldsymbol{y}\left|\mathbb{R}_{\boldsymbol{u} \boldsymbol{v}}\right| \boldsymbol{x}^{\prime}, \boldsymbol{y}^{\prime}\right\rangle\left\langle\boldsymbol{x}^{\prime}, \boldsymbol{z}\left|\mathbb{R}_{\boldsymbol{u} \boldsymbol{w}}\right| \boldsymbol{x}^{\prime \prime}, \boldsymbol{z}^{\prime}\right\rangle\left\langle\boldsymbol{y}^{\prime}, \boldsymbol{z}^{\prime}\left|\mathbb{R}_{\boldsymbol{v} \boldsymbol{w}}\right| \boldsymbol{y}^{\prime \prime}, \boldsymbol{z}^{\prime \prime}\right\rangle \\
& =\int d \boldsymbol{x}^{\prime} d \boldsymbol{y}^{\prime} d \boldsymbol{z}^{\prime}\left\langle\boldsymbol{y}, \boldsymbol{z}\left|\mathbb{R}_{\boldsymbol{v} \boldsymbol{w}}\right| \boldsymbol{y}^{\prime}, \boldsymbol{z}^{\prime}\right\rangle\left\langle\boldsymbol{x}, \boldsymbol{z}^{\prime}\left|\mathbb{R}_{\boldsymbol{u} \boldsymbol{w}}\right| \boldsymbol{x}^{\prime}, \boldsymbol{z}^{\prime \prime}\right\rangle\left\langle\boldsymbol{x}^{\prime}, \boldsymbol{y}^{\prime}\left|\mathbb{R}_{\boldsymbol{u} \boldsymbol{v}}\right| \boldsymbol{x}^{\prime \prime}, \boldsymbol{y}^{\prime \prime}\right\rangle,
\end{aligned}
$$

where the integration measure is defined in (11), and the symbols $\boldsymbol{u}=\left[u, u^{\prime}\right], \boldsymbol{v}=\left[v, v^{\prime}\right]$ and $\boldsymbol{w}=\left[w, w^{\prime}\right]$ stand for the rapidity pairs. With the standard conventions the last equation can be written in an operator form

$$
\mathbb{R}_{\boldsymbol{u v}} \mathbb{R}_{\boldsymbol{u} \boldsymbol{w}} \mathbb{R}_{\boldsymbol{v} \boldsymbol{w}}=\mathbb{R}_{\boldsymbol{v} \boldsymbol{w}} \mathbb{R}_{\boldsymbol{u} \boldsymbol{w}} \mathbb{R}_{\boldsymbol{u} \boldsymbol{v}}
$$

The $R$-matrix (20) was derived as a unique intertwiner for two different sets of Lax operators serving the modular double $[15,16]$ of the $s l(n)$ analog of the quadratic Sklyanin algebra [17,31]. The details of calculations will be published elsewhere.

According to the terminology of the Baxter's book [32], Eq.(21) defines a vertex model. It is easy to see that to within boundary effects the same model can be equivalently reformulated as an interaction-round-a-face (IRF) model. Indeed, the lattice in Fig. 1 can also be formed by periodic translations of a four-edge star (consisting of four edges meeting at the same site), instead of the box diagram of Fig. 3. A little inspection shows that there are only two different types of such 

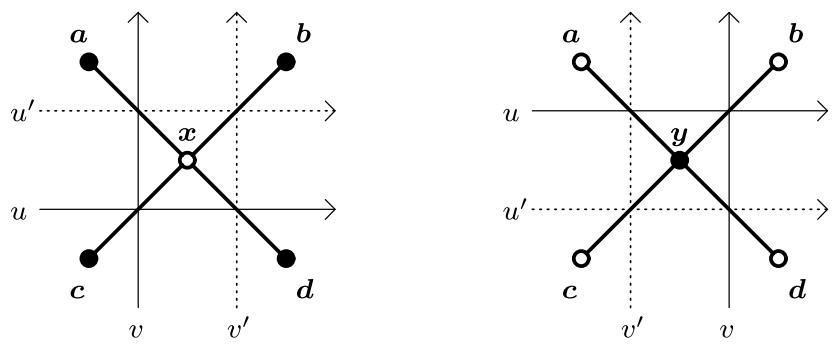

Fig. 4. Two types of four-edge stars: a white-centred star $\mathbb{V}^{(1)}$ (left) and a black-centred $\mathbb{V}^{(2)}$ (right).

stars shown in Fig. 4. Recall that there are two types of sites, shown with open and filled circles. We will refer to them as to "white" or "black" sites, respectively. There are white-centred stars (i.e., centred around a white sites) and black-centred ones. Applying the rules shown in Fig. 2 one can write IRF-type Boltzmann weights corresponding to these stars

$$
\mathbb{V}_{u v}^{(1)}\left(\begin{array}{ll}
\boldsymbol{a} & \boldsymbol{b} \\
\boldsymbol{c} & \boldsymbol{d}
\end{array}\right)=\int d \boldsymbol{x} \overline{\mathbb{W}}_{u-v}(\boldsymbol{c}, \boldsymbol{x}) \overline{\mathbb{W}}_{u^{\prime}-v^{\prime}}(\boldsymbol{b}, \boldsymbol{x}) \mathbb{W}_{u^{\prime}-v}(\boldsymbol{x}, \boldsymbol{a}) \mathbb{W}_{u-v^{\prime}}(\boldsymbol{x}, \boldsymbol{d}),
$$

and

$$
\mathbb{V}_{u v}^{(2)}\left(\begin{array}{ll}
\boldsymbol{a} & \boldsymbol{b} \\
\boldsymbol{c} & \boldsymbol{d}
\end{array}\right)=\int d \boldsymbol{y} \overline{\mathbb{W}}_{u-v}(\boldsymbol{y}, \boldsymbol{b}) \overline{\mathbb{W}}_{u^{\prime}-v^{\prime}}(\boldsymbol{y}, \boldsymbol{c}) \mathbb{W}_{u^{\prime}-v}(\boldsymbol{d}, \boldsymbol{y}) \mathbb{W}_{u-v^{\prime}}(\boldsymbol{a}, \boldsymbol{y}),
$$

where the bold symbols $\boldsymbol{u}$ and $\boldsymbol{v}$ has the same meaning as in (22). The above two expressions are connected by the so-called star-star relation $[19,20]$, which in our case reads

$$
\mathbb{W}_{v^{\prime}-v}(\boldsymbol{d}, \boldsymbol{c}) \mathbb{W}_{u^{\prime}-u}(\boldsymbol{d}, \boldsymbol{b}) \mathbb{V}_{u v}^{(1)}\left(\begin{array}{ll}
\boldsymbol{a} & \boldsymbol{b} \\
\boldsymbol{c} & \boldsymbol{d}
\end{array}\right)=\mathbb{W}_{v^{\prime}-v}(\boldsymbol{b}, \boldsymbol{a}) \mathbb{W}_{u^{\prime}-u}(\boldsymbol{c}, \boldsymbol{a}) \mathbb{V}_{\boldsymbol{u v v}}^{(2)}\left(\begin{array}{ll}
\boldsymbol{a} & \boldsymbol{b} \\
\boldsymbol{c} & \boldsymbol{d}
\end{array}\right)
$$

Apparently this is the simplest relation for the Boltzmann weights which ensures the integrability of the considered model. ${ }^{3}$ Currently, we claim this relation as a conjecture, however, we expect that a complete proof could be obtained by a rather straightforward generalization of the results of [20-24], devoted to discrete-spin models connected with the $s l(n)$ algebra. We have verified this relation in a few orders of perturbation theory in the temperature-like variables (see Appendix B) and made extensive numerical checks for $n=3,4$.

It is worth noting that the Yang-Baxter equation (22) for the composite "box $R$-matrix" (20) is a simple corollary of the star-star relation (26). The same relation also implies yet another Yang-Baxter equation for the IRF-type weights

$$
\begin{aligned}
\int d \boldsymbol{h} \mathbb{V}_{u v}\left(\begin{array}{ll}
c & \boldsymbol{h} \\
\boldsymbol{e} & \boldsymbol{d}
\end{array}\right) \mathbb{V}_{u w}\left(\begin{array}{ll}
\boldsymbol{h} & \boldsymbol{b} \\
\boldsymbol{d} & f
\end{array}\right) \mathbb{V}_{v w}\left(\begin{array}{ll}
\boldsymbol{c} & \boldsymbol{g} \\
\boldsymbol{h} & \boldsymbol{b}
\end{array}\right) \\
=\int d \boldsymbol{a} \mathbb{V}_{v w}\left(\begin{array}{ll}
\boldsymbol{e} & \boldsymbol{a} \\
d & f
\end{array}\right) \mathbb{V}_{u w}\left(\begin{array}{ll}
\boldsymbol{c} & \boldsymbol{g} \\
e & \boldsymbol{a}
\end{array}\right) \mathbb{V}_{u v}\left(\begin{array}{ll}
g & b \\
\boldsymbol{a} & f
\end{array}\right)
\end{aligned}
$$

where

\footnotetext{
3 For $n=2$ the star-star relation (26) is just a consequence of the star-triangle relation, Eq. (1.5) of [13], which is equivalent to the elliptic beta integral [14,29]. However, for $n \geqslant 3$ the corresponding star-triangle relation apparently does not exist (at least it is not known to the authors) and the star-star relation (26) seems to be the simplest relation of this type.
} 


$$
\begin{aligned}
\mathbb{V}_{u v}\left(\begin{array}{ll}
\boldsymbol{a} & \boldsymbol{b} \\
\boldsymbol{c} & \boldsymbol{d}
\end{array}\right) & =\left\{\frac{\mathbb{W}_{v^{\prime}-v}(\boldsymbol{d}, \boldsymbol{c}) \mathbb{W}_{u^{\prime}-u}(\boldsymbol{d}, \boldsymbol{b})}{\mathbb{W}_{v^{\prime}-v}(\boldsymbol{b}, \boldsymbol{a}) \mathbb{W}_{u^{\prime}-u}(\boldsymbol{c}, \boldsymbol{a})}\right\}^{\frac{1}{2}} \mathbb{V}_{\boldsymbol{u v}}^{(1)}\left(\begin{array}{ll}
\boldsymbol{a} & \boldsymbol{b} \\
\boldsymbol{c} & \boldsymbol{d}
\end{array}\right) \\
& =\left\{\frac{\mathbb{W}_{v^{\prime}-v}(\boldsymbol{b}, \boldsymbol{a}) \mathbb{W}_{u^{\prime}-u}(\boldsymbol{c}, \boldsymbol{a})}{\mathbb{W}_{v^{\prime}-v}(\boldsymbol{d}, \boldsymbol{c}) \mathbb{W}_{u^{\prime}-u}(\boldsymbol{d}, \boldsymbol{b})}\right\}^{\frac{1}{2}} \mathbb{V}_{u v}^{(2)}\left(\begin{array}{ll}
\boldsymbol{a} & \boldsymbol{b} \\
\boldsymbol{c} & \boldsymbol{d}
\end{array}\right) .
\end{aligned}
$$

The partition function (21) can be re-written using either of the weights (24), (25) or (28). For example, it easy to see that, up to boundary effects,

$$
Z=\int \prod_{\text {(white stars) }} \mathbb{V}_{\boldsymbol{u} v}\left(\begin{array}{ll}
\boldsymbol{a} & \boldsymbol{b} \\
\boldsymbol{c} & \boldsymbol{d}
\end{array}\right) \prod_{\text {(black sites) }} d \boldsymbol{x},
$$

where the first product is taken over all white-centred stars and $\boldsymbol{a}, \boldsymbol{b}, \boldsymbol{c}, \boldsymbol{d}$ denote the corresponding outer spins, arranged as in Fig. 4. The integral is taken over the spin variables on black internal sites (the integration over the spins on white sites is included through the definitions (28) and (24)). As before, the boundary spins are kept fixed. Note that the weights (24), (25) and (28) differ from each other by equivalence transformations which leave the partition function (29) unchanged (up to boundary contributions).

There are two alternative forms for the RHS in (28), which coincide by virtue of the star-star relation (26). This fact allows one to understand the behaviour of the IRF weight (28) under the complex conjugation. Consider any of the regimes defined in (2) and assume that the rapidity variables obey the relations

$$
u^{*}=u^{\prime}, \quad v^{*}=v^{\prime}, \quad \text { and } \quad 0<\operatorname{Re}\left(u^{\prime}-v^{\prime}, u-v, \ldots\right)<\eta .
$$

Note that these relations allow a homogeneous case $u=u^{\prime}, v=v^{\prime}$, when $u$ and $v$ are real. Taking (30) into account and using (19) one can easily check that the complex conjugation just interchanges the two equivalent forms of (28). It follows then that the IRF weight (28) is real. Further, one can show that there must exist a region for the parameters $p$ and $q$ where this weight is non-negative,

$$
\left[\mathbb{V}_{u v}\left(\begin{array}{ll}
a & b \\
c & d
\end{array}\right)\right]^{*}=\mathbb{V}_{u v}\left(\begin{array}{ll}
a & b \\
c & d
\end{array}\right) \geqslant 0
$$

for all values of the spin variables. First of all note, that in the regimes (2) the single-spin weight (13) is real and non-negative

$$
\mathbb{S}(\boldsymbol{x}) \geqslant 0 .
$$

It vanishes only then at least two components of $\boldsymbol{x}$ coincide. Next, the definition (12) for $\overline{\mathbb{W}}_{\alpha}(\boldsymbol{x}, \boldsymbol{y})$ contains square roots of $\mathbb{S}(\boldsymbol{x})$ and $\mathbb{S}(\boldsymbol{y})$ and therefore there is an ambiguity in choosing signs. However, it is easy to see that these signs cancel out for all internal sites and therefore can be chosen arbitrarily. For definiteness we assume that $\mathbb{S}(\boldsymbol{x})^{\frac{1}{2}}=\left|\mathbb{S}(\boldsymbol{x})^{\frac{1}{2}}\right| \geqslant 0$.

Consider the limit when $p, q \rightarrow 0$ and the ratio $p / q$ is finite. It is convenient to parametrize the rapidity variables as

$$
\begin{array}{rlrl}
u & =\frac{\eta}{2}+\alpha+\frac{\mathrm{i}}{2}(\gamma-\beta), & v & =-\frac{\mathrm{i}}{2}(\gamma+\beta), \\
u^{\prime} & =\frac{\eta}{2}+\alpha-\frac{\mathrm{i}}{2}(\gamma-\beta), & v^{\prime}=+\frac{\mathrm{i}}{2}(\gamma+\beta)
\end{array}
$$

where the variables $\alpha, \beta$ and $\gamma$ are real. Moreover, assume that 


$$
|\operatorname{Re} \alpha| \ll \eta,
$$

which means that $\mathrm{e}^{2 \alpha}=\mathcal{O}(1)$, when $\mathrm{p}, \mathrm{q} \rightarrow 0$. The relations (30) are automatically satisfied. The weights (12) and (13) can be expanded in powers of $p$ and $q$

$$
\begin{aligned}
& \mathbb{W}_{\alpha}(\boldsymbol{x}, \boldsymbol{y})=1+\mathcal{O}(\mathrm{pq})+\cdots \\
& \mathbb{W}_{\eta / 2+\alpha}(\boldsymbol{x}, \boldsymbol{y})=1+\mathcal{O}\left((\mathrm{pq})^{\frac{1}{2}}\right)+\mathcal{O}(\mathrm{pq})+\cdots \\
& \pi^{n-1} n ! \mathbb{S}(\boldsymbol{x}) \prod_{j<k}\left(2 \sin \left(x_{j}-x_{k}\right)\right)^{-2}=1+\mathcal{O}\left(\mathrm{p}^{2}+\mathrm{q}^{2}\right)+\cdots
\end{aligned}
$$

Explicit form of the coefficients to within the forth order is given in Appendix B (the fractional powers arise due to the relation $\mathrm{e}^{-\eta}=(\mathrm{pq})^{\frac{1}{2}}$ ). It is convenient to define sums of exponents of the spin variables, entering the IRF weight (28)

$$
\mathcal{A}_{1}=\sum_{j=1}^{n} \mathrm{e}^{2 \mathrm{i} a_{j}}, \quad \mathcal{B}_{1}=\sum_{j=1}^{n} \mathrm{e}^{2 \mathrm{i} b_{j}}, \quad \mathcal{C}_{1}=\sum_{j=1}^{n} \mathrm{e}^{2 \mathrm{i} c_{j}}, \quad \mathcal{D}_{1}=\sum_{j=1}^{n} \mathrm{e}^{2 \mathrm{i} d_{j}} .
$$

Using the above expansions one obtains

$$
\left|\mathbb{S}(\boldsymbol{b})^{-\frac{1}{2}} \mathbb{S}(\boldsymbol{c})^{-\frac{1}{2}}\right| \mathbb{V}_{\boldsymbol{u} v}\left(\begin{array}{l}
\boldsymbol{a} \boldsymbol{b} \\
\boldsymbol{c d}
\end{array}\right)=1+\mathrm{pq} \mathcal{P}+\mathcal{O}\left((\mathrm{pq})^{\frac{3}{2}}\right)+\cdots
$$

where $\mathcal{P}$ denotes the following expression

$$
\mathcal{P}=\left(\mathrm{e}^{2 \mathrm{i} \beta} \mathcal{A}_{1}+\mathrm{e}^{-2 \mathrm{i} \beta} \mathcal{D}_{1}\right)\left(\mathrm{e}^{2 \mathrm{i} \gamma} \mathcal{B}_{1}^{*}+\mathrm{e}^{-2 \mathrm{i} \gamma} \mathcal{C}_{1}^{*}\right)+\left(\mathrm{e}^{-2 \mathrm{i} \beta} \mathcal{A}_{1}^{*}+\mathrm{e}^{2 \mathrm{i} \beta} \mathcal{D}_{1}^{*}\right)\left(\mathrm{e}^{-2 \mathrm{i} \gamma} \mathcal{B}_{1}+\mathrm{e}^{2 \mathrm{i} \gamma} \mathcal{C}_{1}\right)
$$

which depends on spins and rapidity variables. A few more terms of the expansion is given in Appendix B. They are all manifestly real and thereby confirm the validity of the star-star relation (26) in perturbation theory. Note that the leading term in (37) is strictly positive. The coefficients of the expansions in (35) are analytic functions of the spin variables, thus the integrals over central spins in the star weight (28) are non-singular in every order of the expansion. It follows then that the coefficients in front of powers of $p$ and $q$ in the RHS of (37) are analytic function of the external spins. Therefore, there exists a finite domain of the parameters $p$ and $q$ in the vicinity of $p=q=0$, where the RHS of (37) is strictly positive for all values of the external spins $\boldsymbol{a}, \boldsymbol{b}$, $\boldsymbol{c}, \boldsymbol{d}$. We will call this domain a physical regime. It would certainly be interesting to investigate exact boundaries of this domain.

In the physical regime the considered lattice model becomes a well defined model of statistical mechanics and Euclidean lattice field theory. Using the standard arguments based on the commutativity of transfer matrices and functional equations for the partition function per edge [33-35], we deduced that for a large number of sites $N$ (in the thermodynamic limit) the bulk free energy of the model vanishes,

$$
\lim _{N \rightarrow \infty} N^{-1} \log Z=0,
$$

provided that the normalization (15) in (12) is taken into account. 


\section{Quasi-classical limit}

\subsection{Asymptotics of the Boltzmann weights}

Let us now consider the quasi-classical limit of our model, when one of the nomes is real and fixed, while the other one tends to unity,

$$
\mathrm{q}=\mathrm{e}^{\mathrm{i} \pi \tau}=\text { real, } \quad \mathrm{p}=\mathrm{e}^{\mathrm{i} \pi \sigma} \rightarrow 1, \quad \sigma \rightarrow 0 .
$$

It convenient to use a rescaled parameter

$$
\hbar=-\mathrm{i} \pi \sigma \rightarrow 0,
$$

which plays the role of the Planck constant from the point of view of Euclidean field theory (or the temperature from the point of view of classical statistical mechanics). Introduce a new function

$$
\lambda_{4}(z \mid \tau)=-\mathrm{i} \int_{0}^{z} d w \log \bar{\vartheta}_{4}(z \mid \tau),
$$

where $\bar{\vartheta}_{j}(z \mid \tau)$ is defined in (8). In the limit (40) the elliptic gamma-function (4) and the factor (15) become

$$
\log \Phi(z)=-\frac{1}{\hbar} \lambda_{4}(z \mid \tau) / \hbar+\mathcal{O}\left(\hbar^{0}\right), \quad \log \kappa_{n}(\alpha)=-\frac{1}{\hbar} \lambda_{4}(\operatorname{in} \alpha \mid n \tau)+\mathcal{O}\left(\hbar^{0}\right),
$$

moreover

$$
\begin{aligned}
& \log G(\sigma)=-\frac{\pi^{2}}{12 \hbar}-\frac{1}{2} \log \hbar+\mathcal{O}\left(\hbar^{0}\right), \\
& \log \bar{\vartheta}_{1}(x \mid \sigma)=-\frac{1}{\hbar}\left\{x^{2}-\pi x+\frac{\pi^{2}}{6}\right\}+\mathcal{O}\left(\hbar^{0}\right), \quad 0<x<\pi,
\end{aligned}
$$

where $G(\sigma)$ is defined in (8). In writing quasi-classical expansions of the Boltzmann weights it is convenient to employ the permutation symmetry (18). Below we will assume that components of spin variables are always arranged such that

$$
\boldsymbol{x}=\left(x_{1}, x_{2}, \ldots, x_{n}\right), \quad-\frac{\pi}{2} \leqslant \operatorname{Re}\left(x_{1}\right)<\operatorname{Re}\left(x_{2}\right)<\cdots<\operatorname{Re}\left(x_{n}\right)<\frac{\pi}{2} .
$$

The leading quasi-classical asymptotics of the weights (12) reads

$$
\begin{aligned}
& \log \mathbb{W}_{\alpha}(\boldsymbol{x}, \boldsymbol{y})=-\frac{1}{\hbar} \Lambda_{\alpha}(\boldsymbol{x}, \boldsymbol{y})+\mathcal{O}\left(\hbar^{0}\right), \\
& \log \overline{\mathbb{W}}_{\alpha}(\boldsymbol{x}, \boldsymbol{y})=-\frac{1}{\hbar} \bar{\Lambda}_{\alpha}(\boldsymbol{x}, \boldsymbol{y})-\frac{(n-1)}{2} \log \hbar+\mathcal{O}\left(\hbar^{0}\right),
\end{aligned}
$$

where

$$
\begin{aligned}
& \Lambda_{\alpha}(\boldsymbol{x}, \boldsymbol{y})=-\lambda_{4}(\mathrm{i} n \alpha \mid n \tau)+\sum_{j, k} \lambda_{4}\left(x_{j}-y_{k}+\mathrm{i} \alpha \mid \tau\right), \\
& \bar{\Lambda}_{\alpha}(\boldsymbol{x}, \boldsymbol{y})=\Lambda_{\eta_{0}-\alpha}(\boldsymbol{x}, \boldsymbol{y})+\frac{1}{2} \mathcal{C}(\boldsymbol{x})+\frac{1}{2} \mathcal{C}(\boldsymbol{y}), \quad \eta_{0}=-\mathrm{i} \pi \tau / 2 .
\end{aligned}
$$


Eqs. (48) and (49) are trivial corollaries of the definition (12) and the expansions (43). The function $\mathcal{C}(\boldsymbol{x})$ is determined by the asymptotics of the single-spin weight

$$
\log \mathbb{S}(\boldsymbol{x})=-\frac{1}{\hbar} \mathcal{C}(\boldsymbol{x})-\frac{(n-1)}{2} \log \hbar+\mathcal{O}\left(\hbar^{0}\right),
$$

defined in (13). Using (44) one obtains

$$
\mathcal{C}(\boldsymbol{x})=\frac{\left(n^{2}-1\right) \pi^{2}}{12}+n \sum_{j=1}^{n} x_{j}^{2}-2 \pi \sum_{j=1}^{n} j x_{j}
$$

provided the variables $x_{j}$ are arranged as in (45). Finally, taking into account that $\mathrm{q}$ is assumed real, one deduces that under the complex conjugation

$$
\left(\Lambda_{\alpha}(\boldsymbol{x}, \boldsymbol{y})\right)^{*}=\Lambda_{\alpha^{*}}\left(\boldsymbol{y}^{*}, \boldsymbol{x}^{*}\right),
$$

where $\boldsymbol{x}^{*}=\left(x_{1}^{*}, x_{2}^{*}, \ldots, x_{n}^{*}\right)$ and similarly for $\boldsymbol{y}^{*}$.

\subsection{Classical star-star relation}

Consider now the quasi-classical limit for the composite Boltzmann weights of the stars shown in Fig. 4. To make equations more compact we will write the spin arguments $\boldsymbol{a}, \boldsymbol{b}, \boldsymbol{c}, \boldsymbol{d}$ on the same line, but assume the same spacial arrangement as in Fig. 4. Substituting (47) into (24) and calculating the integral by the saddle point method, one gets

$$
\log \left(\mathbb{V}_{\boldsymbol{u} \boldsymbol{v}}^{(1)}(\boldsymbol{a}, \boldsymbol{b}, \boldsymbol{c}, \boldsymbol{d})\right)=-\frac{1}{\hbar} \mathcal{L}_{\boldsymbol{u}, \boldsymbol{v}}^{(1)}(\boldsymbol{X} \mid \boldsymbol{a}, \boldsymbol{b}, \boldsymbol{c}, \boldsymbol{d})-\frac{(n-1)}{2} \log \hbar+\mathcal{O}\left(\hbar^{0}\right),
$$

where

$$
\mathcal{L}_{\boldsymbol{u}, \boldsymbol{v}}^{(1)}(\boldsymbol{x} \mid \boldsymbol{a}, \boldsymbol{b}, \boldsymbol{c}, \boldsymbol{d})=\bar{\Lambda}_{u-v}(\boldsymbol{c}, \boldsymbol{x})+\bar{\Lambda}_{u^{\prime}-v^{\prime}}(\boldsymbol{b}, \boldsymbol{x})+\Lambda_{u^{\prime}-v}(\boldsymbol{x}, \boldsymbol{a})+\Lambda_{u-v^{\prime}}(\boldsymbol{x}, \boldsymbol{d})
$$

and $\boldsymbol{X}=\left(X_{1}, X_{2}, \ldots, X_{n}\right)$ is the saddle point, determined by the equations

$$
\left.\left(\frac{\partial}{\partial x_{k}}-\frac{\partial}{\partial x_{k+1}}\right) \mathcal{L}_{\boldsymbol{u}, \boldsymbol{v}}^{(1)}(\boldsymbol{x} \mid \boldsymbol{a}, \boldsymbol{b}, \boldsymbol{c}, \boldsymbol{d})\right|_{\boldsymbol{x}=\boldsymbol{X}}=0, \quad k=1,2, \ldots, n-1 .
$$

Remind that $\boldsymbol{x}$ is the multi-component variable, $\boldsymbol{x}=\left(x_{1}, x_{2}, \ldots, x_{n}\right)$, whose components $x_{k}$ are constrained by (10) and cannot be varied independently. That is why there are only $(n-1)$ equations in (55), each containing a difference of two partial derivatives. Define new variables

$$
\begin{array}{ll}
\alpha_{1}=u^{\prime}-v, & \alpha_{2}=\eta_{0}-u^{\prime}+v^{\prime}, \quad \alpha_{3}=\eta_{0}-u+v, \\
\alpha_{4}=u-v^{\prime}, & \alpha_{5}=u^{\prime}-u, \quad \alpha_{6}=v^{\prime}-v,
\end{array}
$$

constrained by three relation

$$
\alpha_{1}+\alpha_{2}+\alpha_{3}+\alpha_{4}=2 \eta_{0}, \quad \alpha_{5}=\alpha_{1}+\alpha_{3}-\eta_{0}, \quad \alpha_{6}=\alpha_{1}+\alpha_{2}-\eta_{0} .
$$

Below we will regard $\alpha_{1}, \alpha_{2}, \alpha_{3}$ as independent variables. Introduce a new function

$$
\begin{aligned}
& \psi\left(X|\boldsymbol{a}, \boldsymbol{b}, \boldsymbol{c}, \boldsymbol{d}| \alpha_{1}, \alpha_{2}, \alpha_{3}, \alpha_{4}\right) \\
& \quad=-\mathrm{i} \sum_{k=1}^{n} \log \frac{\vartheta_{4}\left(X-a_{k}+\mathrm{i} \alpha_{1} \mid \tau\right) \vartheta_{4}\left(X-d_{k}+\mathrm{i} \alpha_{4} \mid \tau\right)}{\vartheta_{4}\left(c_{k}-X+\mathrm{i} \alpha_{3} \mid \tau\right) \vartheta_{4}\left(b_{k}-X+\mathrm{i} \alpha_{2} \mid \tau\right)},
\end{aligned}
$$


which depend on a (single-component) variable $X$ and four multi-component spin variables $\boldsymbol{a}$, $\boldsymbol{b}, \boldsymbol{c}, \boldsymbol{d}$ and the rapidity-type variables $\alpha_{1}, \alpha_{2}, \alpha_{3}, \alpha_{4}$ (it is also implicitly depends on the elliptic modular parameter $\tau$ ). Using (48), (49) and (51) one can write the variational equations (55) in the form

$$
\begin{aligned}
& \psi\left(X_{k+1}|\boldsymbol{a}, \boldsymbol{b}, \boldsymbol{c}, \boldsymbol{d}| \alpha_{1}, \alpha_{2}, \alpha_{3}, \alpha_{4}\right)-\psi\left(X_{k}|\boldsymbol{a}, \boldsymbol{b}, \boldsymbol{c}, \boldsymbol{d}| \alpha_{1}, \alpha_{2}, \alpha_{3}, \alpha_{4}\right) \\
& \quad=+2 \pi-2 n\left(X_{k+1}-X_{k}\right), \quad k=1,2, \ldots, n-1,
\end{aligned}
$$

where the RHS comes from a variation of the function $\mathcal{C}(\boldsymbol{x})$ associated with the central spin in the star (it enters the expression (49)). Similarly, for the second star in Fig. 4, one gets

$$
\log \left(\mathbb{V}_{\boldsymbol{u v}}^{(2)}(\boldsymbol{a}, \boldsymbol{b}, \boldsymbol{c}, \boldsymbol{d})\right)=-\frac{1}{\hbar} \mathcal{L}_{\boldsymbol{u}, \boldsymbol{v}}^{(2)}(\boldsymbol{Y} \mid \boldsymbol{a}, \boldsymbol{b}, \boldsymbol{c}, \boldsymbol{d})-\frac{(n-1)}{2} \log \hbar+\mathcal{O}\left(\hbar^{0}\right)
$$

where

$$
\mathcal{L}_{\boldsymbol{u}, \boldsymbol{v}}^{(2)}(\boldsymbol{y} \mid \boldsymbol{a}, \boldsymbol{b}, \boldsymbol{c}, \boldsymbol{d})=\bar{\Lambda}_{u-v}(\boldsymbol{y}, \boldsymbol{b})+\bar{\Lambda}_{u^{\prime}-v^{\prime}}(\boldsymbol{y}, \boldsymbol{c})+\Lambda_{u^{\prime}-v}(\boldsymbol{d}, \boldsymbol{y})+\Lambda_{u-v^{\prime}}(\boldsymbol{a}, \boldsymbol{y}),
$$

and the saddle point $\boldsymbol{Y}=\left(Y_{1}, Y_{2}, \ldots, Y_{n}\right)$ is determined by the variational equations ${ }^{4}$

$$
\begin{aligned}
& \psi\left(Y_{k+1}|\boldsymbol{a}, \boldsymbol{b}, \boldsymbol{c}, \boldsymbol{d}|-\alpha_{4},-\alpha_{3},-\alpha_{2},-\alpha_{1}\right)-\psi\left(Y_{k}|\boldsymbol{a}, \boldsymbol{b}, \boldsymbol{c}, \boldsymbol{d}|-\alpha_{4},-\alpha_{3},-\alpha_{2},-\alpha_{1}\right) \\
& \quad=-2 \pi+2 n\left(Y_{k+1}-Y_{k}\right), \quad k=1,2, \ldots, n-1 .
\end{aligned}
$$

The quasi-classical expansion of the quantum star-star relation (26) leads an infinite number of non-trivial relations - one relation for each order of the expansion in $\hbar$. In the leading order one obtains,

$$
\mathcal{L}_{u, v}^{(1)}(X \mid a, b, c, d)-\Delta_{u, v}(a, b, c, d)=\mathcal{L}_{u, v}^{(2)}(Y \mid a, b, c, d)+\Delta_{u, v}(a, b, c, d),
$$

where $\boldsymbol{X}$ and $\boldsymbol{Y}$ are determined by (59) and (62) and

$$
\Delta_{u, v}(\boldsymbol{a}, \boldsymbol{b}, \boldsymbol{c}, \boldsymbol{d})=\frac{1}{2}\left(\Lambda_{v^{\prime}-v}(\boldsymbol{b}, \boldsymbol{a})+\Lambda_{u^{\prime}-u}(\boldsymbol{c}, \boldsymbol{a})-\Lambda_{v^{\prime}-v}(\boldsymbol{d}, \boldsymbol{c})-\Lambda_{u^{\prime}-u}(\boldsymbol{d}, \boldsymbol{b})\right) .
$$

We name the formula (63) a classical star-star relation.

For the weight function (28) one obtains

$$
\log \mathbb{V}_{\boldsymbol{u} \boldsymbol{v}}(\boldsymbol{a}, \boldsymbol{b}, \boldsymbol{c}, \boldsymbol{d})=-\frac{1}{\hbar} \mathcal{L}_{\boldsymbol{u}, \boldsymbol{v}}(\boldsymbol{a}, \boldsymbol{b}, \boldsymbol{c}, \boldsymbol{d})-\frac{(n-1)}{2} \log \hbar+\mathcal{O}(\hbar),
$$

where the Lagrangian density $\mathcal{L}_{\boldsymbol{u}, \boldsymbol{v}}(\boldsymbol{a}, \boldsymbol{b}, \boldsymbol{c}, \boldsymbol{d})$ coincides with the LHS (or the RHS) of the (63). Writing it in full, one obtains

$$
\begin{aligned}
\mathcal{L}_{\boldsymbol{u}, \boldsymbol{v}}(\boldsymbol{a}, \boldsymbol{b}, \boldsymbol{c}, \boldsymbol{d}) \\
\quad=\bar{\Lambda}_{u-v}(\boldsymbol{c}, \boldsymbol{X})+\bar{\Lambda}_{u^{\prime}-v^{\prime}}(\boldsymbol{b}, \boldsymbol{X})+\Lambda_{u^{\prime}-v}(\boldsymbol{X}, \boldsymbol{a})+\Lambda_{u-v^{\prime}}(\boldsymbol{X}, \boldsymbol{d})-\Delta_{\boldsymbol{u}, \boldsymbol{v}}(\boldsymbol{a}, \boldsymbol{b}, \boldsymbol{c}, \boldsymbol{d}) \\
=\bar{\Lambda}_{u-v}(\boldsymbol{Y}, \boldsymbol{b})+\bar{\Lambda}_{u^{\prime}-v^{\prime}}(\boldsymbol{Y}, \boldsymbol{c})+\Lambda_{u^{\prime}-v}(\boldsymbol{d}, \boldsymbol{Y})+\Lambda_{u-v^{\prime}}(\boldsymbol{a}, \boldsymbol{Y})+\Delta_{\boldsymbol{u}, \boldsymbol{v}}(\boldsymbol{a}, \boldsymbol{b}, \boldsymbol{c}, \boldsymbol{d}),
\end{aligned}
$$

where $\boldsymbol{X}$ and $\boldsymbol{Y}$ are determined by the variational equations (59) and (62).

Consider now the regime (30) with real $q$ as in (40). Assume that the spins $\boldsymbol{a}, \boldsymbol{b}, \boldsymbol{c}, \boldsymbol{d}$ are real. Then one can show that if $\boldsymbol{X}$ solves (59) then $\boldsymbol{Y}=\boldsymbol{X}^{*}$ solves (62), where the star denotes the complex conjugation. Having this in mind and using (52) it is easy to check that the complex

\footnotetext{
4 Notice different signs in the RHS of this equation with respect to (59).
} 


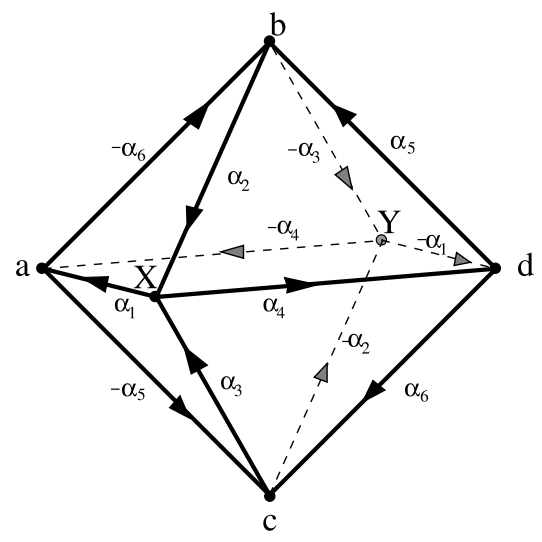

Fig. 5. Graphical representation of the classical star-star relation (68).

conjugation just interchanges two alternative expressions in (66). This means that the Lagrangian density $\mathcal{L}_{\boldsymbol{u}, \boldsymbol{v}}(\boldsymbol{a}, \boldsymbol{b}, \boldsymbol{c}, \boldsymbol{d})$ is real.

From (12) and (47) it follows that

$$
\Lambda_{\alpha}(\boldsymbol{a}, \boldsymbol{b})+\Lambda_{-\alpha}(\boldsymbol{b}, \boldsymbol{a})=0 .
$$

Using this equality together with (49), substituting (54), (61) and (64) into the classical star-star relation (63) and moving all terms there to one side, one obtains

$$
\begin{aligned}
& \Lambda_{\alpha_{1}}(\boldsymbol{X}, \boldsymbol{a})+\Lambda_{\alpha_{2}}(\boldsymbol{b}, \boldsymbol{X})+\Lambda_{\alpha_{3}}(\boldsymbol{c}, \boldsymbol{X})+\Lambda_{\alpha_{4}}(\boldsymbol{X}, \boldsymbol{d})+\Lambda_{\alpha_{6}}(\boldsymbol{d}, \boldsymbol{c})+\Lambda_{\alpha_{5}}(\boldsymbol{d}, \boldsymbol{b}) \\
& \quad+\Lambda_{-\alpha_{4}}(\boldsymbol{Y}, \boldsymbol{a})+\Lambda_{-\alpha_{3}}(\boldsymbol{b}, \boldsymbol{Y})+\Lambda_{-\alpha_{2}}(\boldsymbol{c}, \boldsymbol{Y})+\Lambda_{-\alpha_{1}}(\boldsymbol{Y}, \boldsymbol{d})+\Lambda_{-\alpha_{6}}(\boldsymbol{a}, \boldsymbol{b}) \\
& \quad+\Lambda_{-\alpha_{5}}(\boldsymbol{a}, \boldsymbol{c})+\mathcal{C}(\boldsymbol{X})-\mathcal{C}(\boldsymbol{Y})=0 .
\end{aligned}
$$

The terms of this relation can be conveniently associated with edges and vertices of an octahedron. Consider a perfect octahedron shown in Fig. 5. Its edges are oriented, as indicated by arrows, such that there are exactly two incoming and two outgoing edges at each vertex. The vertices are assigned to the spin variables $\boldsymbol{a}, \boldsymbol{b}, \boldsymbol{c}, \boldsymbol{d}, \boldsymbol{X}, \boldsymbol{Y}$, as indicated. The edges carry rapiditytype variables $\pm \alpha_{1}, \pm \alpha_{2}, \ldots, \pm \alpha_{6}$, shown near edges. A perfect octahedron contains six pairs of parallel edges. The values of the $\alpha$-variables on parallel edges are equal in absolute value, but differ in signs. Moreover, the $\alpha$-variables are constrained by the relations (57). Each edge corresponds to a $\Lambda$-term in (68). For example, the edge directed from $\boldsymbol{X}$ to $\boldsymbol{a}$ and carrying the rapidity label $\alpha_{1}$ in Fig. 5 corresponds to the term $\Lambda_{\alpha_{1}}(\boldsymbol{X}, \boldsymbol{a})$ in (68). Similarly for all other edges. Further, according to (57), the arithmetic sum of the $\alpha$-variables on four edges meeting at the vertex $\boldsymbol{X}$ (regardless their directions) is equal to $+2 \pi$. This vertex contributes the term " $+\mathcal{C}(\boldsymbol{X})$ " to (68). Similarly, the sum of $\alpha$ 's at the vertex $\boldsymbol{Y}$ is equal to $-2 \pi$. This vertex contributes the term " $-\mathcal{C}(\boldsymbol{Y})$ ". Finally, thanks to (57) the sum of $\alpha$ 's for each of the remaining four vertices $\boldsymbol{a}$, $\boldsymbol{b}, \boldsymbol{c}, \boldsymbol{d}$ exactly vanishes. These vertices do not contribute any $\mathcal{C}$-terms into (68).

From mathematical point of view it is convenient to regard Eqs. (59) and (62) as constrains placed on six spin variables at the vertices of the octahedron. Interestingly, these constrains can be re-written in two other equivalent forms. Differentiating (68) with respect to the spins $\boldsymbol{a}$ and $\boldsymbol{d}$ one obtains 


$$
\left\{\begin{array}{l}
\psi\left(a_{k+1}|\boldsymbol{b}, \boldsymbol{X}, \boldsymbol{Y}, \boldsymbol{c}|-\alpha_{6},+\alpha_{1},-\alpha_{4},-\alpha_{5}\right) \\
\quad-\psi\left(a_{k}|\boldsymbol{b}, \boldsymbol{X}, \boldsymbol{Y}, \boldsymbol{c}|-\alpha_{6},+\alpha_{1},-\alpha_{4},-\alpha_{5}\right)=0, \\
\psi\left(d_{k+1}|\boldsymbol{b}, \boldsymbol{X}, \boldsymbol{Y}, \boldsymbol{c}|+\alpha_{5},+\alpha_{4},-\alpha_{1},+\alpha_{6}\right) \\
\quad-\psi\left(d_{k}|\boldsymbol{b}, \boldsymbol{X}, \boldsymbol{Y}, \boldsymbol{c}|+\alpha_{5},+\alpha_{4},-\alpha_{1},+\alpha_{6}\right)=0,
\end{array}\right.
$$

where $k=1,2, \ldots, n-1$. Here we have used the fact that the LHS of (68) is stationary with respect to variations of $\boldsymbol{X}$ and $\boldsymbol{Y}$ by virtue of (59) and (62). Similarly, differentiating (68) with respect to the spins $\boldsymbol{b}$ and $\boldsymbol{c}$ one obtains

$$
\left\{\begin{array}{l}
\psi\left(b_{k+1}|\boldsymbol{X}, \boldsymbol{a}, \boldsymbol{d}, \boldsymbol{Y}|+\alpha_{2},-\alpha_{6},+\alpha_{5},-\alpha_{3}\right) \\
\quad-\psi\left(b_{k}|\boldsymbol{X}, \boldsymbol{a}, \boldsymbol{d}, \boldsymbol{Y}|+\alpha_{2},-\alpha_{6},+\alpha_{5},-\alpha_{3}\right)=0, \\
\psi\left(c_{k+1}|\boldsymbol{X}, \boldsymbol{a}, \boldsymbol{d}, \boldsymbol{Y}|+\alpha_{3},-\alpha_{5},+\alpha_{6},-\alpha_{2}\right) \\
\quad-\psi\left(c_{k}|\boldsymbol{X}, \boldsymbol{a}, \boldsymbol{d}, \boldsymbol{Y}|+\alpha_{3},-\alpha_{5},+\alpha_{6},-\alpha_{2}\right)=0,
\end{array}\right.
$$

where $k=1,2, \ldots, n-1$.

\subsection{Discrete non-linear equations}

In the limit (41) the partition function (29) develops a typical quasi-classical asymptotics. Substituting (65) into (29) and calculating the integral by the saddle point method one obtains,

$$
\log Z=-\frac{1}{\hbar} \mathcal{A}(\{\boldsymbol{y}\})+\mathcal{O}\left(\hbar^{0}\right)
$$

where the action

$$
\mathcal{A}(\{\boldsymbol{y}\})=\sum_{\text {white stars }} \mathcal{L}_{\boldsymbol{u}, \boldsymbol{v}}(\boldsymbol{a}, \boldsymbol{b}, \boldsymbol{c}, \boldsymbol{d})
$$

where the sum over all white-centred stars and $\boldsymbol{a}, \boldsymbol{b}, \boldsymbol{c}, \boldsymbol{d}$ denote the corresponding outer spins arranged as in Fig. 4. Here $\{\boldsymbol{y}\}$ denotes an equilibrium configuration of spins on the black sublattice, which is determined by the Euler-Lagrange equations

$$
\frac{\delta}{\delta \boldsymbol{y}(\vec{r})} \mathcal{A}(\{\boldsymbol{y}\})=0
$$

where $\vec{r}$ is an integer two-dimensional vector numerating the internal sites on the black sublattice (remind that the spins are varied subject to the constraints (10)). Using the first expression from (66) one could bring these equations to the form

$$
\frac{\delta}{\delta \boldsymbol{y}} \mathcal{L}_{\boldsymbol{u}, \boldsymbol{v}}^{(2)}(\boldsymbol{y} \mid \boldsymbol{a}, \boldsymbol{b}, \boldsymbol{c}, \boldsymbol{d})=0
$$

for every black site. The function $\mathcal{L}_{\boldsymbol{u}, \boldsymbol{v}}^{(2)}(\boldsymbol{y} \mid \boldsymbol{a}, \boldsymbol{b}, \boldsymbol{c}, \boldsymbol{d})$ is defined in (61) and the symbols $\boldsymbol{y}, \boldsymbol{a}, \boldsymbol{b}, \boldsymbol{c}$, $\boldsymbol{d}$ denote the spins on a (black-centred) star, arranged as in Fig. 4 on the right side. Eqs. (74) are explicitly presented in (62) in an expanded form. Further, the definition (66) for the Lagrangian $\mathcal{L}_{\boldsymbol{u}, \boldsymbol{v}}(\boldsymbol{a}, \boldsymbol{b}, \boldsymbol{c}, \boldsymbol{d})$, entering the action (72), involves the variational equations (55),

$$
\frac{\delta}{\delta \boldsymbol{x}} \mathcal{L}_{\boldsymbol{u}, \boldsymbol{v}}^{(1)}(\boldsymbol{x} \mid \boldsymbol{a}, \boldsymbol{b}, \boldsymbol{c}, \boldsymbol{d})=0
$$

for every white site. These equations are explicitly presented in (59). Combining Eqs. (74) and (75) (respectively, their expanded forms (59), (62)), one gets a system of non-linear difference equations for all internal sites of the lattice (the boundary spins are fixed). Note, that each of 
these equations involves variables on five sites, belonging to a four-edge star. These classical integrable equations can be regarded as a generalization of the Laplace-type system associated with the Adler-Bobenko-Suris $Q_{4}$ equation $[13,26]$ to the case of multi-component field variables. Their integrability is inherited from the integrability of the quantum model. It could also be independently established from the classical star-star relation (63) (in particular, the latter implies the existence of an infinite set of local integrals of motion).

It appears that for Dirichlet (fixed) boundary conditions solutions of the classical equation (59), (62) possess the following property. We expect that in the bulk of a large lattice (i.e. away from the boundary) all solutions converge to some constant solution, independent of the boundary values of the spins. According to (39) it is reasonable to expect that the action (72) evaluated on this constant solution should vanish. The corresponding solution could be easily found. Assume that all spins on the lattice are equal to same vector

$$
\boldsymbol{x}^{(c)}=\left(x_{1}, x_{2}, \ldots, x_{n}\right), \quad x_{j}=\frac{\pi}{n}\left(j-\frac{n+1}{2}\right), j=1,2, \ldots, n .
$$

Then it is easy to check that all equations (59), (62) are satisfied and that

$$
\Lambda_{\alpha}\left(\boldsymbol{x}^{(c)}, \boldsymbol{x}^{(c)}\right)=\mathcal{C}\left(\boldsymbol{x}^{(c)}\right)=0 .
$$

Therefore the action (72) exactly vanishes on this solution in complete agreement with (39). It would be interesting to verify whether this it is an absolute minimum of the action and, more generally, to study its global convexity properties.

As an illustration consider the case $n=2$. The two-component spin variables in this case contain only one independent continuous variable, so that it will be more convenient to use the scalar argument $x$, assuming that the corresponding two-component spin is equal to $x=(-x, x)$, and similarly for all other spins. Eqs. (48) and (51) simplify to

$$
\Lambda_{\alpha}(x, y)=-\mathrm{i} \int_{0}^{x-y} d w \log \frac{\vartheta_{4}(w+\mathrm{i} \alpha \mid \tau)}{\vartheta_{4}(w-\mathrm{i} \alpha \mid \tau)}-\mathrm{i} \int_{\pi / 2}^{x+y} d w \log \frac{\vartheta_{4}(w+\mathrm{i} \alpha \mid \tau)}{\vartheta_{4}(w-\mathrm{i} \alpha \mid \tau)}
$$

and

$$
\mathcal{C}(x)=\frac{\pi^{2}}{4}+4 x^{2}-2 \pi|x|, \quad-\frac{\pi}{2} \leqslant x \leqslant \frac{\pi}{2} .
$$

Note that for $n=2$ the function $\Lambda_{\alpha}(x, y)$ becomes symmetric upon interchanging $x$ and $y$. Using the above expressions in (49) and (66) one can bring the action (72) to the form

$$
\mathcal{A}\left(\left\{x_{s}\right\}\right)=\sum_{\langle r s\rangle} \Lambda_{\alpha_{r s}}\left(x_{r}, x_{s}\right)+\sum_{s} \mathcal{C}\left(x_{s}\right)
$$

where the first sum is over all edges $\langle r s\rangle$, while the second sum is over all sites $s$. The variables $\alpha_{r s}$ take one of the four values $\alpha_{1}, \alpha_{2}, \alpha_{3}, \alpha_{4}$, defined in (56), depending on the type of the edge $\langle r s\rangle$ and the values of rapidity variables passing through the edge. The variational equations (75) and (59) for the white-centred stars, as in Fig. 4, reduce to

$$
\varphi_{\alpha_{1}}(x, a)+\varphi_{\alpha_{2}}(x, b)+\varphi_{\alpha_{3}}(x, c)+\varphi_{\alpha_{4}}(x, d)=2 \pi-8 x
$$

where

$$
\varphi_{\alpha}(x, y)=\partial_{x} \Lambda_{a}(x, y)=\frac{1}{\mathrm{i}} \log \frac{\vartheta_{4}(x-y+\mathrm{i} \alpha) \vartheta_{4}(x+y+\mathrm{i} \alpha)}{\vartheta_{4}(x-y-\mathrm{i} \alpha) \vartheta_{4}(x+y-\mathrm{i} \alpha)} .
$$


Similarly, for the black-centred stars one gets

$$
\varphi_{\alpha_{4}}(y, a)+\varphi_{\alpha_{3}}(y, b)+\varphi_{\alpha_{2}}(y, c)+\varphi_{\alpha_{1}}(y, d)=2 \pi-8 y
$$

where the spins are arranged as in Fig. 4. The constant solution (76) in this case reads

$$
x^{(c)}=\frac{\pi}{4},
$$

for all sites of the lattice. Observing that

$$
\Lambda_{\alpha}\left(\frac{\pi}{4}, \frac{\pi}{4}\right)=\mathcal{C}\left(\frac{\pi}{4}\right)=0
$$

one easily concludes that action (80) on this solution vanishes exactly $\mathcal{A}\left(\left\{x_{j}^{(c)}\right\}\right)=0$. Let us show that this is the minimum of the action. ${ }^{5}$ Expanding the latter in the vicinity of this solution

$$
x_{s}=\frac{\pi}{4}+\varepsilon_{s}, \quad \varepsilon_{s} \rightarrow 0,
$$

one gets

$$
\mathcal{A}\left(\left\{x_{s}\right\}\right)=\sum_{\langle r s\rangle}\left(\zeta_{4}\left(\mathrm{i} \alpha_{r s}\right)-\zeta_{3}\left(\mathrm{i} \alpha_{r s}\right)\right)\left(\varepsilon_{r}-\varepsilon_{s}\right)^{2}+2 c \sum_{s} \varepsilon_{s}^{2}+\mathcal{O}\left(\varepsilon^{3}\right),
$$

where

$$
\zeta_{k}(z)=\frac{1}{\mathrm{i}} \frac{\partial}{\partial z} \log \vartheta_{k}(z \mid \tau), \quad k=3,4,
$$

and

$$
c=2+\zeta_{3}\left(\mathrm{i} \alpha_{1}\right)+\zeta_{3}\left(\mathrm{i} \alpha_{2}\right)+\zeta_{3}\left(\mathrm{i} \alpha_{3}\right)+\zeta_{3}\left(\mathrm{i} \alpha_{4}\right) .
$$

Remind that $\tau$ is assumed to be purely imaginary, $\operatorname{Im} \tau>0$. Using the inequality

$$
\zeta_{4}(\mathrm{i} \alpha)>0>\zeta_{3}(\mathrm{i} \alpha)>-\frac{2 \alpha}{\pi|\tau|}, \quad 0<\alpha<\pi|\tau| / 2
$$

and remembering that the sum of $\alpha$ 's is constrained by (57), with $\eta_{0}=-\mathrm{i} \pi \tau / 2$, one can check that

$$
c>0, \quad 0<\alpha_{k}<\eta_{0}, \quad k=1,2,3,4,
$$

and thus that (87) defines a positive-definite quadratic form. The constant solution (84) could be though as an analog of the isoradial solution of the Hirota equations describing planar circle patterns $[11,36]$.

\footnotetext{
5 Since the expression (79) is non-analytic for $x=0$, one needs to check the case where all spins are vanishing $x_{S}=0$. Careful considerations show that the value of the action for this configuration is greater than zero.
} 


\section{Conclusion}

We formulated a new solvable edge-interaction model of statistical mechanics with multicomponent continuous spin variables taking values on a circle. The Boltzmann weights are given by Eqs. (12), (13). The weights satisfy the star-star relation (26), which ensures the integrability of the model. Currently, we claim this relation as a conjecture. We have verified this relation in a few orders of perturbation theory in the temperature-like variables (see Appendix B) and made extensive numerical checks for $n=3,4$. We expect that a complete proof could be obtained by a rather straightforward generalization of the results of [20-24], devoted to discrete-spin models connected with the $s l(n)$ algebra.

The star-star relation (26) implies the validity of the Yang-Baxter equations in the vertex (22) and IRF (27) forms. The partition function per edge of the lattice is calculated exactly in the thermodynamic limit by means of the inversion relations [33-35]. The result is included into the normalization of the Boltzmann weights given by (14), (15). With this normalization the bulk free energy of model vanishes, see (39).

In Section 4 we study the quasi-classical (or the low-temperature) limit when one of the elliptic nomes tends to unity, $p \rightarrow 1$, and obtain the classical version (Eqs. (63) and (68)) of the star-star relation.

Here we have only formulated our model on a simple square lattice, but the latter can be replaced with a rather general planar graph. The required construction is similar to that described in [13] with some modifications accommodating the two types of rapidity lines shown in Fig. 2. The partition function then will be invariant under certain deformations of the planar graph connected with the star-star (26) and inversion (17) relations. This property is known as Baxter's $Z$-invariance [37]. Evidently, this property continues to hold in quasi-classical limit. As a result the action of the classical system (72) evaluated on the stationary configuration will be invariant under the "star-star" transformations connected with the classical star-star relation (68). These moves are similar to the "star-triangular" transformations for the classical action introduced in [10]. Remind that, geometrically, the classical star-triangle relation can be associated with a tetrahedron formed by a set of six face diagonals of a cube. By this reason the star-triangular moves are often referred to as "cubic flips" of faces of a quadrilateral surface [38]. From this point of view the star-star relation (68) could be associated with "rhombic-dodecahedral flips" of quadrilateral surfaces, since the octahedron in Fig. 5, representing the various terms in (68), forms a set of face diagonals of a rhombic dodecahedron.

As mentioned in the introduction, for $n=2$ the model provides a master solution [13] of the Yang-Baxter equation, which contains all known edge-interaction models with singlecomponent spins as particular cases. It turns out that for $n>2$ the model also possesses a similar "master" property. The Boltzmann weights (12) allow a large number of interesting limiting cases. For example, one could consider a low-temperature limit when one of the nomes tends to a root of unity,

$$
\mathrm{p} \rightarrow \mathrm{e}^{\mathrm{i} \pi / N}, \quad N=2,3,4, \ldots
$$

This limit is similar, but more complicated than the quasi-classical limit considered in Section 4. In this case one obtains a "hybrid" model which couples a classical integrable system, involving continuous spin variables, and an Ising-type model of statistical mechanics with discrete multicomponent spins variable taking values in $\left(\mathbb{Z}_{N}\right)^{n-1}$. The Boltzmann weights are expressed with elliptic theta functions. In general, the emerging model is spatially inhomogeneous, since its 
Boltzmann weights depend on solutions of the classical equation of motion. ${ }^{6}$ In a particular homogeneous case connected to a constant classical solution (similar to (76)) this model reduces to the $s l_{n}$-generalization [24] of the Kashiwara-Miwa model [2]. In the trigonometric limit this elliptic model further reduces to the $s l_{n}$-generalized chiral Potts model [21,22], which is in its turn equivalent to the $N$-state Zamolodchikov-Bazhanov-Baxter model [20,39] on a cubic lattice (the number $n$ becomes the size of the lattice in the "hidden" third dimension). We hope to consider all these limits and connections in the future (the $n=2$ case has been thoroughly studied in [13]). It would also be interesting to explore possible connections of our results to the discretespin models considered in [40].

\section{Note added in proof}

The star-star relation (26) can be equivalently reduced to the $A_{n}$-type integral transformation of elliptic hypergeometric integrals obtained by Rains [41] (see Theorem 4.1 therein). The authors are grateful to Hjalmar Rosengren who brough our attention to this work.

\section{Acknowledgements}

The authors thank R.J. Baxter, A.I. Bobenko, V.V. Mangazeev, V.P. Spiridonov and Y.B. Suris for interesting discussions. This work was partially supported by the Australian Research Council.

\section{Appendix A. Inversion relation}

Theorem 11 from [16] (related to $A_{n-1}$ root system) can be re-written in our notations as

$$
\begin{aligned}
\int d \boldsymbol{y} & \overline{\mathbb{W}}_{\alpha}(\boldsymbol{x}, \boldsymbol{y}) \overline{\mathbb{W}}_{\beta}(\boldsymbol{y}, \boldsymbol{z}) \prod_{j=1}^{n} \Phi\left(\sigma-y_{j}+\mathrm{i} n \alpha\right) \Phi\left(y_{j}-\sigma+\mathrm{i} n \beta\right) \\
= & \overline{\mathbb{W}}_{\alpha+\beta}(\boldsymbol{x}, \boldsymbol{z}) \frac{\kappa_{n}(\eta-\alpha-\beta) \Phi(\mathrm{i} \eta-\mathrm{i} n \alpha) \Phi(\mathrm{i} \eta-\mathrm{i} n \beta)}{\Phi(\mathrm{i} \eta-\mathrm{i} n(\alpha+\beta)) \kappa_{n}(\eta-\alpha) \kappa_{n}(\eta-\beta)} \\
& \times \prod_{j=1}^{n} \Phi\left(\sigma-x_{j}+\mathrm{i} \alpha\right) \Phi\left(z_{j}-\sigma+\mathrm{i} \beta\right) \Phi\left(x_{j}-\sigma+\mathrm{i}(n \beta-\alpha)\right) \\
& \times \Phi\left(\sigma-z_{j}+\mathrm{i}(n \alpha-\beta)\right) .
\end{aligned}
$$

Taking the limit $\beta \rightarrow-\alpha$ and using (16) and

$$
\Phi(z) \Phi(-z)=1, \quad \lim _{\epsilon \rightarrow 0} \frac{\Phi(\mathrm{i} \eta-\mathrm{i} n \epsilon)}{\kappa_{n}(\eta-\epsilon)}=1, \quad \overline{\mathbb{W}}_{0}(\boldsymbol{x}, \boldsymbol{z})=\frac{1}{n !} \sum_{\hat{\sigma}} \delta(\boldsymbol{x}, \hat{\sigma}(\boldsymbol{z})),
$$

one obtains the inversion relation (17).

\footnotetext{
6 These classical equation of motion are very similar to Eqs. (75) and (74). Conceptually the hybrid model described here is very much similar to a model of quantum field theory on a non-trivial classical background.
} 


\section{Appendix B. Series expansions of in powers of $p$ and $q$}

Define power sums of exponents of the spin variables, entering the star weight (24),

$$
\mathcal{A}_{k}=\sum_{j=1}^{n} \mathrm{e}^{2 \mathrm{i} k a_{j}}, \quad \mathcal{B}_{k}=\sum_{j} \mathrm{e}^{2 \mathrm{i} k b_{j}}, \quad \ldots, \quad \mathcal{X}_{k}=\sum_{j=1}^{n} \mathrm{e}^{2 \mathrm{i} k x_{j}}, \quad k=1,2, \ldots
$$

Consider the physical regime (2), (30). Then rapidity variables can be parametrized as

$$
\begin{aligned}
u & =\frac{\eta}{2}+\alpha+\frac{\mathrm{i}}{2}(\gamma-\beta), & v & =-\frac{\mathrm{i}}{2}(\gamma+\beta), \\
u^{\prime} & =\frac{\eta}{2}+\alpha-\frac{\mathrm{i}}{2}(\gamma-\beta), & v^{\prime} & =+\frac{\mathrm{i}}{2}(\gamma+\beta)
\end{aligned}
$$

where the parameters $\mathrm{s} \alpha, \beta$ and $\gamma$ are real. Moreover, assume

$$
|\operatorname{Re} \alpha| \ll \eta, \quad \mathrm{p} \sim \mathrm{q} \rightarrow 0,
$$

Using the product expression (4) for the elliptic gamma-function it is not difficult to obtain the following expansions for the Boltzmann weights (12) and (13),

$$
\begin{aligned}
\kappa_{n}(\eta / 2+\alpha) \mathbb{W}_{\eta / 2+\alpha}(\boldsymbol{a}, \boldsymbol{b}) \\
=1+\frac{1}{2}(\mathrm{pq})^{\frac{1}{2}} \mathrm{e}^{2 \alpha} \mathcal{A}_{1}^{*} \mathcal{B}_{1}+\mathrm{pqe}^{4 \alpha}\left\{\left(\mathcal{A}_{1}^{*}\right)^{2}\left(\mathcal{B}_{1}\right)^{2}+\mathcal{A}_{2}^{*} \mathcal{B}_{2}\right\} \\
\quad+\frac{1}{6}(\mathrm{pq})^{\frac{3}{2}} \mathrm{e}^{6 \alpha}\left\{\left(\mathcal{A}_{1}^{*}\right)^{3}\left(\mathcal{B}_{1}\right)^{3}+2 \mathcal{A}_{3}^{*} \mathcal{B}_{3}+3 \mathcal{A}_{1}^{*} \mathcal{A}_{2}^{*} \mathcal{B}_{1} \mathcal{B}_{2}\right\} \\
\quad+(\mathrm{pq})^{\frac{1}{2}}\left(\mathrm{p}^{2}+\mathrm{q}^{2}\right) \mathrm{e}^{2 \alpha} \mathcal{A}_{1}^{*} \mathcal{B}_{1}-(\mathrm{pq})^{\frac{3}{2}} \mathrm{e}^{-2 \alpha} \mathcal{A}_{1} \mathcal{B}_{1}^{*} \\
+\frac{1}{24} \mathrm{p}^{2} \mathrm{q}^{2} \mathrm{e}^{8 \alpha}\left\{\left(\mathcal{A}_{1}^{*}\right)^{4}\left(\mathcal{B}_{1}\right)^{4}+6\left(\mathcal{A}_{1}^{*}\right)^{2} \mathcal{A}_{2}^{*}\left(\mathcal{B}_{1}\right)^{2} \mathcal{B}_{2}\right. \\
\left.+3\left(\mathcal{A}_{2}^{*}\right)^{2}\left(\mathcal{B}_{2}\right)^{2}+8 \mathcal{A}_{1}^{*} \mathcal{A}_{3}^{*} \mathcal{B}_{1} \mathcal{B}_{3}+6 \mathcal{A}_{4}^{*} \mathcal{B}_{4}\right\} \\
\quad+\mathrm{pq}\left(\mathrm{p}^{2}+\mathrm{q}^{2}\right) \mathrm{e}^{4 \alpha}\left(\mathcal{A}_{1}^{*}\right)^{2}\left(\mathcal{B}_{1}\right)^{2}-\mathrm{p}^{2} \mathrm{q}^{2} \mathcal{A}_{1}^{*} \mathcal{A}_{1} \mathcal{B}_{1}^{*} \mathcal{B}_{1}+\mathcal{O}\left(\mathrm{p}^{5}\right),
\end{aligned}
$$

where the order of the correction terms here and below is shown for $p \sim q$,

$$
\begin{aligned}
\kappa_{n}(\alpha) \mathbb{W}_{\alpha}(\boldsymbol{a}, \boldsymbol{b})= & 1+\mathrm{pq}\left(1+\mathrm{p}^{2}+\mathrm{q}^{2}\right)\left\{\mathrm{e}^{2 \alpha} \mathcal{A}_{1}^{*} \mathcal{B}_{1}-\mathrm{e}^{-2 \alpha} \mathcal{A}_{1} \mathcal{B}_{1}^{*}\right\}-\mathrm{p}^{2} \mathrm{q}^{2} \mathcal{A}_{1}^{*} \mathcal{A}_{1} \mathcal{B}_{1}^{*} \mathcal{B}_{1} \\
& +\mathrm{p}^{2} \mathrm{q}^{2}\left\{\cosh 4 \alpha\left(\mathcal{A}_{1}^{*}\right)^{2}\left(\mathcal{B}_{1}\right)^{2}+\sinh 4 \alpha \mathcal{A}_{2} \mathcal{B}_{2}^{*}\right\}+\mathcal{O}\left(\mathrm{p}^{6}\right),
\end{aligned}
$$

and

$$
\begin{aligned}
\pi^{n-1} n ! S(x) \prod_{j<k}\left(2 \sin \left(x_{j}-x_{k}\right)\right)^{-2} \\
=1-\left(\mathrm{p}^{2}+\mathrm{q}^{2}\right)\left\{\mathcal{X}_{1}^{*} \mathcal{X}_{1}-1\right\}+\mathrm{p}^{2} \mathrm{q}^{2}\left\{\mathcal{X}_{1}^{*} \mathcal{X}_{1}-1\right\}^{2} \\
\quad+\frac{1}{2}\left(\mathrm{p}^{4}+\mathrm{q}^{4}\right)\left\{4-4 \mathcal{X}_{1}^{*} \mathcal{X}_{1}+\left(\mathcal{X}_{1}^{*}\right)^{2}\left(\mathcal{X}_{1}\right)^{2}-\mathcal{X}_{2}^{*} \mathcal{X}_{2}\right\}+\mathcal{O}\left(\mathrm{p}^{6}\right) .
\end{aligned}
$$

From (15) one obtains

$$
\kappa(\eta / 2+\alpha)=1+(\mathrm{pq})^{\frac{n}{2}} \mathrm{e}^{2 n \alpha}+\mathcal{O}\left(\mathrm{p}^{n+2}\right) .
$$


The definition of the IRF weight (28) requires an evaluation of integrals over the central spin in the star. The results depend on the value of $n$. Below we restrict ourselves to the case $n=3$. For any function $f(\boldsymbol{x})$ of the spin variable $\boldsymbol{x}$ define the following integral

$$
\mathcal{J}[f(\boldsymbol{x})]=\frac{1}{\pi^{n-1} n !} \int\left(\prod_{j<k}\left(2 \sin \left(x_{j}-x_{k}\right)\right)^{2}\right) f(\boldsymbol{x}) d \boldsymbol{x}, \quad d \boldsymbol{x}=d x_{1} d x_{2} \cdots d x_{n-1} .
$$

Below we will need the following integrals

$$
\begin{aligned}
& \mathcal{J}[1]=\mathcal{J}\left[\mathcal{X}_{1} \mathcal{X}_{1}^{*}\right]=1, \\
& \mathcal{J}\left[\left(\mathcal{X}_{1}\right)^{2}\left(\mathcal{X}_{1}^{*}\right)^{2}\right]=\mathcal{J}\left[\mathcal{X}_{2} \mathcal{X}_{2}^{*}\right]=2,
\end{aligned}
$$

which are given for a general $n$ and specific integrals

$$
\begin{aligned}
& \mathcal{J}\left[\left(\mathcal{X}_{1}\right)^{3}\right]=\mathcal{J}\left[\left(\mathcal{X}_{1}^{*}\right)^{3}\right]=1, \\
& \mathcal{J}\left[\mathcal{X}_{1} \mathcal{X}_{2}\right]=\mathcal{J}\left[\mathcal{X}_{1}^{*} \mathcal{X}_{2}^{*}\right]=-1,
\end{aligned}
$$

valid for $n=3$. Using the above expansions one obtains for the IRF weight for $n=3$ (28)

$$
\begin{aligned}
|\mathbb{S}(\boldsymbol{c}) \mathbb{S}(\boldsymbol{b})|^{-\frac{1}{2}} \mathbb{V}_{\boldsymbol{u} \boldsymbol{v}}\left(\begin{array}{ll}
\boldsymbol{a} & \boldsymbol{b} \\
\boldsymbol{c} & \boldsymbol{d}
\end{array}\right) \\
=1+\mathrm{pq} \mathcal{P}+(\mathrm{pq})^{\frac{3}{2}} \mathcal{Q}+\mathrm{p}^{2} \mathrm{q}^{2}\left(1+\frac{1}{8} \mathcal{P}^{2}+\frac{1}{4} \mathcal{R}-\mathcal{S}\right) \\
\quad+\frac{1}{2} \mathrm{pq}\left(\mathrm{p}^{2}+\mathrm{q}^{2}\right) \mathcal{P}+\mathcal{O}\left(\mathrm{p}^{5}\right)
\end{aligned}
$$

where we used the following notations

$$
\begin{aligned}
& \mathcal{P}=\left(\mathrm{e}^{2 \mathrm{i} \beta} \mathcal{A}_{1}+\mathrm{e}^{-2 \mathrm{i} \beta} \mathcal{D}_{1}\right)\left(\mathrm{e}^{2 \mathrm{i} \gamma} \mathcal{B}_{1}^{*}+\mathrm{e}^{-2 \mathrm{i} \gamma} \mathcal{C}_{1}^{*}\right)+\left(\mathrm{e}^{-2 \mathrm{i} \beta} \mathcal{A}_{1}^{*}+\mathrm{e}^{2 \mathrm{i} \beta} \mathcal{D}_{1}^{*}\right)\left(\mathrm{e}^{-2 \mathrm{i} \gamma} \mathcal{B}_{1}+\mathrm{e}^{2 \mathrm{i} \gamma} \mathcal{C}_{1}\right), \\
& \mathcal{Q}=\mathrm{e}^{6 \alpha}\left(\mathrm{e}^{-2 \mathrm{i} \beta} \mathcal{A}_{1} \mathcal{D}_{1}^{*}+\mathrm{e}^{2 \mathrm{i} \beta} \mathcal{A}_{1}^{*} \mathcal{D}_{1}\right)+\mathrm{e}^{-6 \alpha}\left(\mathrm{e}^{2 \mathrm{i} \gamma} \mathcal{B}_{1} \mathcal{C}_{1}^{*}+\mathrm{e}^{-2 \mathrm{i} \gamma} \mathcal{B}_{1}^{*} \mathcal{C}_{1}\right), \\
& \mathcal{R}=\left(\mathrm{e}^{4 \mathrm{i} \beta} \mathcal{A}_{2}+\mathrm{e}^{-4 \mathrm{i} \beta} \mathcal{D}_{2}\right)\left(\mathrm{e}^{4 \mathrm{i} \gamma} \mathcal{B}_{2}^{*}+\mathrm{e}^{-4 \mathrm{i} \gamma} \mathcal{C}_{2}^{*}\right)+\left(\mathrm{e}^{-4 \mathrm{i} \beta} \mathcal{A}_{2}^{*}+\mathrm{e}^{4 \mathrm{i} \beta} \mathcal{D}_{2}^{*}\right)\left(\mathrm{e}^{-4 \mathrm{i} \gamma} \mathcal{B}_{2}+\mathrm{e}^{4 \mathrm{i} \gamma} \mathcal{C}_{2}\right), \\
& \mathcal{S}=\left(\mathrm{e}^{2 \mathrm{i} \beta} \mathcal{A}_{1}+\mathrm{e}^{-2 \mathrm{i} \beta} \mathcal{D}_{1}\right)\left(\mathrm{e}^{-2 \mathrm{i} \beta} \mathcal{A}_{1}^{*}+\mathrm{e}^{2 \mathrm{i} \beta} \mathcal{D}_{1}^{*}\right)+\left(\mathrm{e}^{-2 \mathrm{i} \gamma} \mathcal{B}_{1}+\mathrm{e}^{2 \mathrm{i} \gamma} \mathcal{C}_{1}\right)\left(\mathrm{e}^{2 \mathrm{i} \gamma} \mathcal{B}_{1}^{*}+\mathrm{e}^{-2 \mathrm{i} \gamma} \mathcal{C}_{1}^{*}\right)
\end{aligned}
$$

All additional integrals (beyond (B.9) and (B.10)), required for (B.11) in the given order, exactly vanish. Note all terms in (B.11) are manifestly real and thereby confirm the validity of the starstar relation (26) in perturbation theory.

\section{References}

[1] L. Onsager, Crystal statistics. I. A two-dimensional model with an order-disorder transition, Phys. Rev. 65 (1944) 117-149.

[2] M. Kashiwara, T. Miwa, A class of elliptic solutions to the star-triangle relation, Nucl. Phys. B 275 (1986) $121-134$.

[3] G. von Gehlen, V. Rittenberg, $Z(n)$-symmetric quantum chains with an infinite set of conserved charges and $Z(n)$ zero modes, Nucl. Phys. B 257 (1985) 351.

[4] H. Au-Yang, B.M. McCoy, J.H.H. Perk, S. Tang, M.-L. Yan, Commuting transfer matrices in the chiral Potts models: Solutions of star-triangle equations with genus > 1, Phys. Lett. A 123 (1987) 219-223. 
[5] R.J. Baxter, J.H.H. Perk, H. Au-Yang, New solutions of the star-triangle relations for the chiral Potts model, Phys. Lett. A 128 (1988) 138-142.

[6] V.A. Fateev, A.B. Zamolodchikov, Self-dual solutions of the star-triangle relations in $Z_{N}$-models, Phys. Lett. A 92 (1) (1982) 37-39.

[7] R.J. Baxter, A rapidity-independent parameter in the star-triangle relation, in: MathPhys Odyssey, 2001, in: Prog. Math. Phys., vol. 23, Birkhäuser, Boston, MA, 2002, pp. 49-63.

[8] A.B. Zamolodchikov, "Fishing-net" diagrams as a completely integrable system, Phys. Lett. B 97 (1) (1980) 63-66.

[9] A.Y. Volkov, L.D. Faddeev, Yang-Baxterization of the quantum dilogarithm, Zap. Nauchn. Sem. POMI 224 (1995) 146-154. English translation in: J. Math. Sci. 88 (1998) 202-207.

[10] V.V. Bazhanov, V.V. Mangazeev, S.M. Sergeev, Faddeev-Volkov solution of the Yang-Baxter equation and discrete conformal symmetry, Nucl. Phys. B 784 (2007) 234-258, arXiv:hep-th/0703041.

[11] A.I. Bobenko, B.A. Springborn, Variational principles for circle patterns and Koebe's theorem, Trans. Amer. Math. Soc. 365 (2004) 659-689.

[12] K. Stephenson, Introduction to Circle Packing. The Theory of Discrete Analytic Functions, Cambridge University Press, Cambridge, 2005.

[13] V.V. Bazhanov, S.M. Sergeev, A master solution of the quantum Yang-Baxter equation and classical discrete integrable equations, arXiv:1006.0651 [math-ph].

[14] V.P. Spiridonov, Elliptic beta integrals and solvable models of statistical mechanics, arXiv:1011.3798 [hep-th].

[15] L. Faddeev, Modular double of a quantum group, in: Conférence Moshé Flato 1999, vol. I (Dijon), in: Math. Phys. Stud., vol. 21, Kluwer Acad. Publ., Dordrecht, 2000, pp. 149-156, arXiv:math/9912078.

[16] V.P. Spiridonov, Essays on the theory of elliptic hypergeometric functions, Uspekhi Mat. Nauk 63 (3) (2008) 3-72.

[17] E.K. Sklyanin, Some algebraic structures connected with the Yang-Baxter equation, Funct. Anal. Appl. 16 (4) (1982) 263-270.

[18] R.J. Baxter, Partition function of the eight-vertex lattice model, Ann. Phys. 70 (1972) 193-228.

[19] R.J. Baxter, The Yang-Baxter equations and the Zamolodchikov model, Physica D 18 (1986) 321-347.

[20] V.V. Bazhanov, R.J. Baxter, New solvable lattice models in three-dimensions, J. Statist. Phys. 69 (1992) $453-585$.

[21] V.V. Bazhanov, R.M. Kashaev, V.V. Mangazeev, Y.G. Stroganov, $\left(\mathbb{Z}_{N}\right)^{n-1}$ generalization of the chiral Potts model, Comm. Math. Phys. 138 (1991) 393-408.

[22] E. Date, M. Jimbo, K. Miki, T. Miwa, Generalized chiral Potts models and minimal cyclic representations of $U_{q}(g l(n, C))$, Comm. Math. Phys. 137 (1991) 133-148.

[23] R.M. Kashaev, V.V. Mangazeev, T. Nakanishi, Yang-Baxter equation for the $s l(n)$ chiral Potts model, Nucl. Phys. B 362 (1991) 563-582.

[24] S.M. Sergeev, $Z_{N}^{\otimes n}$-broken model, Preprint IHEP 92-07, Protvino, 1992.

[25] V.E. Adler, A.I. Bobenko, Y.B. Suris, Classification of integrable equations on quad-graphs. The consistency approach, Comm. Math. Phys. 233 (3) (2003) 513-543.

[26] V.E. Adler, Y.B. Suris, $Q_{4}$ : integrable master equation related to an elliptic curve, Int. Math. Res. Not. 47 (2004) 2523-2553.

[27] S.N.M. Ruijsenaars, First order analytic difference equations and integrable quantum systems, J. Math. Phys. 38 (2) (1997) 1069-1146.

[28] G. Felder, A. Varchenko, The elliptic gamma function and $S L(3, Z) \ltimes Z^{3}$, Adv. Math. 156 (1) (2000) 44-76.

[29] V.P. Spiridonov, On the elliptic beta function, Uspekhi Mat. Nauk 56 (1) (2001) 181-182. English translation in: Russ. Math. Surveys 56 (1) (2001) 185-186.

[30] E. Whittaker, G. Watson, A Course of Modern Analysis, Cambridge University Press, Cambridge, 1927.

[31] A.A. Belavin, Dynamical symmetry of integrable quantum systems, Nucl. Phys. B 180 (1981) 189-200.

[32] R.J. Baxter, Exactly Solved Models in Statistical Mechanics, Academic, London, 1982.

[33] Y.G. Stroganov, A new calculation method for partition functions in some lattice models, Phys. Lett. A 74 (1-2) (1979) 116-118.

[34] A.B. Zamolodchikov, $Z_{4}$-symmetric factorized $S$-matrix in two space-time dimensions, Comm. Math. Phys. 69 (2) (1979) $165-178$.

[35] R.J. Baxter, The inversion relation method for some two-dimensional exactly solved models in lattice statistics, J. Statist. Phys. 28 (1) (1982) 1-41.

[36] A.I. Bobenko, C. Mercat, Y.B. Suris, Linear and nonlinear theories of discrete analytic functions. Integrable structure and isomonodromic Green's function, J. Reine Angew. Math. 583 (2005) 117-161.

[37] R.J. Baxter, Solvable eight-vertex model on an arbitrary planar lattice, Philos. Trans. Roy. Soc. London Ser. A 289 (1359) (1978) 315-346.

[38] A.I. Bobenko, Y.B. Suris, On the Lagrangian structure of integrable quad-equations, Lett. Math. Phys. 92 (2010) 17-31, arXiv:0912.2464 [nlin.SI]. 
[39] A.B. Zamolodchikov, Tetrahedron equations and the relativistic S matrix of straight strings in $(2+1)$-dimensions, Comm. Math. Phys. 79 (1981) 489-505.

[40] A. Zabrodin, Intertwining operators for Sklyanin algebra and elliptic hypergeometric series, arXiv:1012.1228 [math$\mathrm{ph}]$.

[41] E.M. Rains, Transformations of elliptic hypergeometric integrals, Ann. Math. 171 (1) (2004) 169-243. 